Copyright by the American Physical Society. Howard, M ; Frojdh, P ; Lauritsen, KB, Jan 2000. systems with nonequilibrium phase transitions," PHYSICAL REVIEW E 61(1): 167-183. DOI: 10.1103/PhysRevE.61.167.

\title{
Surface critical behavior in systems with nonequilibrium phase transitions
}

\author{
Martin Howard, ${ }^{1, *}$ Per Fröjdh, ${ }^{2, \dagger}$ and Kent Bækgaard Lauritsen $3,4, \uparrow$ \\ ${ }^{1}$ Department of Physics, Virginia Tech, Blacksburg, Virginia 24061-0435 \\ ${ }^{2}$ Department of Physics, Stockholm University, Box 6730, S-113 85 Stockholm, Sweden \\ ${ }^{3}$ Center for Chaos and Turbulence Studies, Niels Bohr Institute, DK-2100 Copenhagen, Denmark \\ ${ }^{4}$ Danish Meteorological Institute, Atmosphere Ionosphere Research Division, DK-2100 Copenhagen, Denmark
}

(Received 29 July 1999)

\begin{abstract}
We study the surface critical behavior of branching-annihilating random walks with an even number of offspring (BARW) and directed percolation (DP) using a variety of theoretical techniques. Above the upper critical dimensions $d_{c}$, with $d_{c}=4$ (DP) and $d_{c}=2$ (BARW), we use mean field-theory to analyze the surface phase diagrams using the standard classification into ordinary, special, surface, and extraordinary transitions. For the case of BARW, at or below the upper critical dimension $d \leqslant d_{c}$, we use field theoretic methods to study the effects of fluctuations. As in the bulk, the field-theory suffers from technical difficulties associated with the presence of a second critical dimension. However, we are still able to analyze the phase diagrams for BARW in $d=1$ and 2, which turn out to be very different from their mean field analog. Furthermore, for the case of BARW only (and not for DP), we find two independent surface $\beta_{1}$ exponents in $d=1$, arising from two distinct definitions of the order parameter. Using an exact duality transformation on a lattice BARW model in $d=1$, we uncover a relationship between these two surface $\beta_{1}$ exponents at the ordinary and special transitions. Many of our predictions are supported using Monte Carlo simulations of two different models belonging to the BARW universality class.
\end{abstract}

PACS number(s): 05.40.-a, 64.60.Ak, 64.60.Ht

\section{INTRODUCTION}

The study of surface critical behavior in equilibrium statistical mechanics has established the importance of boundaries in critical systems and their impact on scaling and universality [1]. Quantities measured close to the surface can scale differently than in the bulk, and can possess distinct critical surface exponents. Depending on the boundary conditions, various surface universality classes are possible each with different values for the surface exponents. In this paper we will be interested in the surface critical behavior of certain dynamic systems, which possess a nonequilibrium phase transition from an active into an absorbing state from which the system cannot escape.

The most prominent example of a system with an absorbing state is directed percolation (DP). It describes the directed growth of a cluster governed by a growth probability $p$ of its fundamental constituents. For probabilities below a critical value, $p<p_{c}$, the cluster will die after a finite time, which means that the system becomes trapped in the vacuum - the unique empty state. On the other hand, for high enough growth probabilities $p>p_{c}$, there is a finite probability that the cluster will always remain active. Exactly at $p$ $=p_{c}$, there is a critical phase transition from the active into the absorbing state [2]. A whole range of other systems possessing a phase transition from a nontrivial active phase into a unique absorbing state fall into this universality class.

\footnotetext{
*Present address: Department of Physics, Simon Fraser University, Burnaby, British Columbia, Canada V5A 1S6. Electronic address: mjhoward@sfu.ca

†Electronic address: frojdh@physto.se

\#Electronic address: baekgard@nbi.dk
}

Some examples include epidemics, chemical reactions, catalysis, and the contact process (see Ref. [3] and references therein).

During the last few years, however, studies have also been carried out for systems with absorbing states which do not belong to the DP class. For instance, the model of branching-annihilating random walks with an even number of offspring (BARW) exhibits quite different behavior [4-6], and defines a separate universality class. Other models in this class (at least in $d=1$ ) include certain probabilistic cellular automata [7], monomer-dimer models [8-10], nonequilibrium kinetic Ising models [11], and generalized DP with two absorbing states (DP2) [12]. These models escape from the DP universality class by possessing an extra conservation law or symmetry: for the BARW model, a "parity" conservation of the total number of particles modulo 2 ; for the other models, an underlying symmetry between their absorbing states.

In the present paper we study the impact of surfaces on the critical behavior of the DP and BARW models. Previous work has concentrated on surface effects in DP using field theoretic methods [13,14], Monte Carlo simulations in $d$ $=1$ and 2 [14-16], the density matrix renormalization group in $d=1$ [17], and series expansions in $d=1[18,19]$. Relations between surface DP and local persistence probabilities were explored in Ref. [20]. Work has also been performed on active, but slanted, walls in DP, which give rise to a "curtain" of activity whose width is given by an angle-dependent correction to bulk DP [21]. Critical surface effects for a model in the BARW universality class were first studied in Ref. [22]. In this paper we will build on this earlier work by presenting a unified picture of surface critical behavior of both BARW and DP. After summarizing the main details of DP and BARW in the bulk, we present a comprehensive 
analysis of the surface critical behavior of both models using mean field theory. This involves the usual classification into ordinary, special, surface, and extraordinary transitions. However, below their respective upper critical dimensions, fluctuation effects become important in both models, and this leads to the breakdown of mean field theory. In order to understand this fluctuation regime we employ a variety of theoretical techniques. First, we construct a phenomenological scaling theory which is able to describe the various surface universality classes. This scaling theory for BARW can then, to some extent, be justified using field-theoretic techniques (a field theory for surface DP was already presented in Ref. [13]). However, the BARW field theory suffers from technical problems associated with the presence of a second critical dimension, which means that the interesting $d=1$ regime cannot be accessed satisfactorily. Nevertheless some results can be derived field theoretically which we put together to draw up a $(1+1)$-dimensional surface phase diagram. This phase diagram displays many differences from its mean field analog. In addition, using exact techniques involving a mapping to a quantum spin Hamiltonian, we have been able to establish an exact duality transformation for a lattice BARW model in $d=1$. We find that this links together two of the boundary phase transitions in $d=1$ in a nontrivial way (as suggested in Ref. [22]). We have also performed extensive Monte Carlo simulations for BARW and DP2 which support many of our theoretical conclusions.

The paper is organized as follows: in Sec. II we briefly introduce the bulk DP and BARW models. Then in Sec. III we consider the surface behavior of DP, where we present an extensive mean field analysis. We also summarize details of the fluctuation regime for $d<d_{c}=4$. In Secs. IV A and IV B, we give the phase diagram for surface BARW in mean field theory and in $d=1$, respectively. These results can be contained within a scaling theory presented in Sec. IV C. We then discuss in Sec. IV D how this analysis can be partially justified using field theoretic methods. In Sec. IV E, we give some exact results for $d=1$. Our theoretical analysis is then backed up using computer simulations of the lattice models introduced in Sec. V. Details of these simulations are presented in Sec. VI. Finally, in Sec. VII we round off with some conclusions.

\section{BULK DP AND BARW}

We begin by briefly reviewing the definitions of the DP and BARW models. The update rules for bond DP in $d+1$ dimensions on a tilted square lattice are easily defined: for each site at time $t$, form bonds with probability $p$ to the neighboring sites at time $t+1$ [2]. An example of a cluster grown from a single seed according to these rules is shown in Fig. 1(a).

For growth probabilities below a certain threshold such a process will eventually die out, whereas for higher values there is a finite probability of survival, which means that the system is in the active state [23]. As is well known [24-26], various reaction-diffusion models also fall into the DP universality class. The simplest of these is defined by the fol-

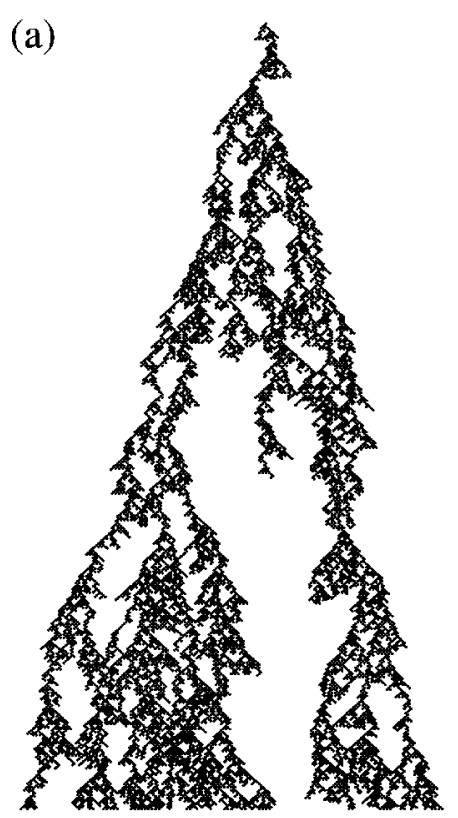

(b)

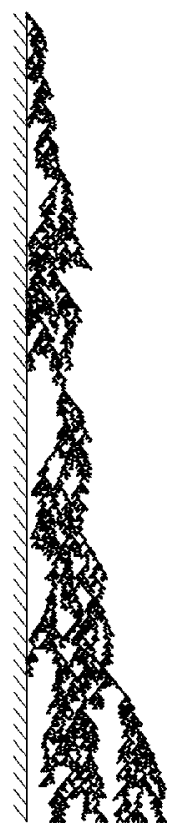

FIG. 1. DP clusters grown from a single seed (a) in the bulk and (b) next to a wall.

lowing reaction scheme for a single species of diffusing particles:

$$
\begin{gathered}
A \rightarrow A+A \quad \text { with rate } \sigma, \\
A+A \rightarrow A \quad \text { with rate } \lambda, \\
A \rightarrow \varnothing \text { with rate } \mu,
\end{gathered}
$$

where, in the corresponding lattice model, we allow for multiple (bosonic) occupancy of any given site.

The second system which we will analyze in detail is the BARW model [4-6]. This is defined again by a (bosonic) particle model, with the following reaction processes:

$$
\begin{gathered}
A \rightarrow(m+1) A \quad \text { with rate } \sigma_{m}, \\
A+A \rightarrow \varnothing \quad \text { with rate } \lambda .
\end{gathered}
$$

For $m$ odd, the above model is known to belong to the DP universality class; however, for $m$ even, we have a new universality class. Unless otherwise specified when we refer to the BARW model we will be referring to the even $m$ case.

The growth of both BARW and DP clusters in the bulk close to criticality can be summarized by a set of independent exponents. A natural choice is to consider $\nu_{\perp}$ and $\nu_{\|}$, which describe the divergence of the correlation lengths in space, $\xi_{\perp} \sim|\Delta|^{-\nu_{\perp}}$, and time, $\xi_{\|} \sim|\Delta|^{-\nu} \|_{\|}$. Here the parameter $\Delta$ describes the deviation from the critical point (in mean field theory $\Delta=\mu-\sigma$ for DP, but $\Delta=-m \sigma_{m}$ for BARW). We also need the order parameter exponent $\beta$, which can be defined in two a priori different ways: it is either governed by the percolation probability (the probability that a cluster grown from a finite seed never dies),

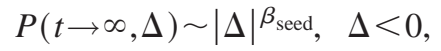



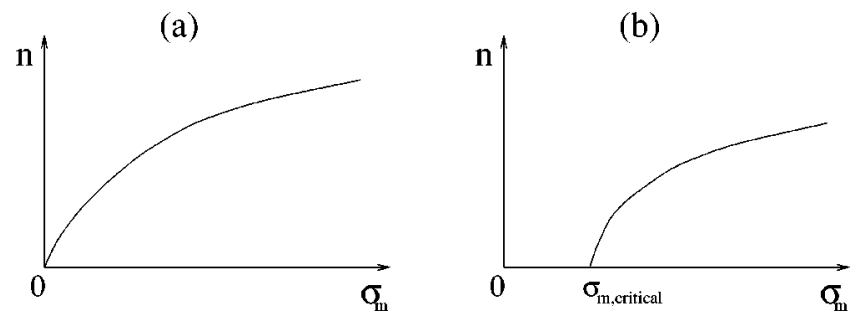

FIG. 2. Schematic bulk behavior for BARW of the density $n$ as a function of the branching rate $\sigma_{m}$ for (a) $d \geqslant 2$ and (b) $d=1$.

or by the coarse-grained density of active sites in the steady state,

$$
n(\Delta) \sim|\Delta|^{\beta_{\text {dens }}, \quad \Delta<0 .}
$$

When $\Delta<0$ the system is said to be in an active state, whereas for $\Delta=0$ the system is critical (with an algebraically decaying density), and for $\Delta>0$ (if applicable) the system is inactive (with an exponentially decaying density) [27]. For the case of DP, it is known that $\beta$ is unique: $\beta_{\text {seed }}=\beta_{\text {dens }}$ in any dimension, both above and below the upper critical dimension $d_{c}=4$. This follows from fieldtheoretic considerations [23,24], and has been verified by extensive numerical work. The relation also holds for BARW in $1+1$ dimensions, a result first suggested by numerics and now backed up by an exact duality mapping [28]. However, this exponent equality is certainly not always true: if we consider the BARW mean field regime valid for spatial dimensions $d>d_{c}=2$, then the system is in a critical inactive state only for a zero branching rate, where the density decays away as a power law. However, any nonzero branching rate results in an active state, with a nonzero steady state density [see Fig. 2(a)] [6]. This density [Eq. (4)] approaches zero continuously (as the branching rate is reduced toward zero) with the mean field exponent $\beta_{\text {dens }}=1$. Nevertheless, for $d$ $>2$, the survival probability [Eq. (3)] of a particle cluster will be finite for any value of the branching rate, implying that $\beta_{\text {seed }}=0$ in mean field theory. This result follows from the non-recurrence of random walks in $d>2$.

Field theoretically, DP is believed to be satisfactorily understood - the appropriate field theory (sometimes called Reggeon field theory) [24,26] is well under control and the exponents have been computed to two loop order in an $\epsilon$ $=4-d$ expansion [25]. However, for the case of BARW, a description of the $(1+1)$-dimensional case poses considerable difficulties for the field theory [6]. These stem from the presence of two critical dimensions: $d_{c}=2$ (above which mean field theory applies) and $d_{c}^{\prime} \approx 4 / 3$. For $d>d_{c}^{\prime}$ the behavior of Fig. 2(a) holds, i.e., an active state results for any nonzero value of the branching $\sigma_{m}$, whereas for $d<d_{c}^{\prime}$ the system is only active for $\sigma_{m}>\sigma_{m \text {, critical }}$, as shown in Fig. 2(b) [6]. This means that the physical spatial dimension $d$ $=1$ cannot be accessed using epsilon expansions down from the upper critical dimension $d_{c}=2$. Furthermore, for the $\sigma_{m}<\sigma_{m \text {,critical }}$ region, the system is not inactive (in the sense of an exponentially decaying density). Instead this entire phase is controlled by the annihilation fixed point of the $A$ $+A \rightarrow \varnothing$ process, where the density decays away as a power law. Hence this phase should rather be considered as still being critical.

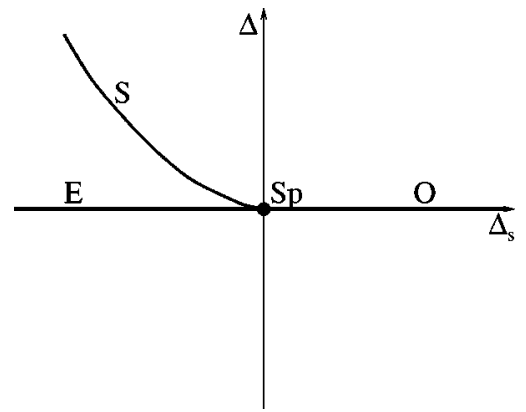

FIG. 3. Schematic mean field phase diagram for surface DP. See text for an explanation of the labeling.

Despite the problems associated with BARW for $d<d_{c}^{\prime}$, we can still put forward a general scaling theory for DP and BARW, valid both above and below their critical dimensions. However, we must retain a possible distinction between $\beta_{\text {seed }}$ and $\beta_{\text {dens }}$. For example, the average lifetime $\langle t\rangle$ of finite clusters can be derived from the scaling form for the survival probability

$$
P(t, \Delta)=|\Delta|^{\beta_{\text {seed }} \varphi\left(t / \xi_{\|}\right) .}
$$

We then find $\langle t\rangle \sim|\Delta|^{-\tau}$, where $\tau=\nu_{\|}-\beta_{\text {seed }}$. The appropriate scaling form for the density $n(\mathbf{x}, t)$, given that the cluster was started at $\mathbf{x}=\mathbf{0}$ and $t=0$, is

$$
n(x, t, \Delta)=|\Delta|^{\beta_{\text {seed }}+\beta_{\text {dens }} f\left(x / \xi_{\perp}, t / \xi_{\|}\right) .}
$$

Notice that rotational symmetry about the seeding point $\mathbf{x}$ $=\mathbf{0}$ implies that the spatial coordinates enter the scaling function only as $x=|\mathbf{x}|$, the distance from the seeding point. Using expression (6) we see that the average mass of finite clusters, $\langle s\rangle \sim|\Delta|^{-\gamma}$, is related to the other exponents via the following hyperscaling relation

$$
\nu_{\|}+d \nu_{\perp}=\beta_{\text {seed }}+\beta_{\text {dens }}+\gamma .
$$

Note that Eq. (7) is consistent with the distinct upper critical dimensions for BARW and DP. Using the above mean field values for BARW and $\nu_{\perp}=1 / 2, \nu_{\|}=1$, and $\gamma=1$, we verify $d_{c}=2$. In contrast, for DP one has the mean field exponents $\beta_{\mathrm{dens}}=\beta_{\mathrm{seed}}=1$ and $d_{c}=4$.

\section{SURFACE DP}

We now briefly review the surface critical behavior of DP, and indicate how the above relations and exponents are modified in a semi-infinite geometry, where we place a wall at $x_{\perp}=0\left[\mathbf{x}=\left(\mathbf{x}_{\|}, x_{\perp}\right)\right.$, with the $\perp$ and $\|$ directions being relative to the wall]. An example of such a cluster grown close to a wall is shown in Fig. 1(b).

A schematic phase diagram for surface DP is shown in Fig. 3 (see Ref. [13]), where $\Delta_{s}$ is the deviation of the surface from criticality. In Fig. 3, the labeling conforms to the standard nomenclature of surface critical phenomena: $\mathrm{O}$ stands for the ordinary transition (bulk critical, surface inactive); $\mathrm{Sp}$ is for the special transition (bulk and surface both critical); $\mathrm{S}$ is for the surface transition (surface critical, bulk inactive); and finally $\mathrm{E}$ stands for the extraordinary transition (surface active, bulk critical). 
The bulk exponents are, of course, unchanged by the presence of a surface and, furthermore, one can show that the correlation length exponents on the surface are also the same as in the bulk. Hence, except at the special transition, one finds just one extra exponent: the surface density exponent $\beta_{1 \text {,dens }}$. This is defined from the steady-state density at the wall. For example, at the ordinary transition, we have

$$
n\left(x_{\perp}=0, \Delta\right) \sim|\Delta|^{\beta_{1, \text { dens }}^{\mathrm{O}}, \quad \Delta<0 .}
$$

On the other hand, at the multicritical special transition, one finds two independent surface exponents - a new surface density exponent, $\beta_{1 \text {,dens }}^{\mathrm{Sp}}$, and a crossover exponent $\phi_{1}$. In principle one could also allow for a second type of surface $\beta_{1}$ exponent, one defined from a survival probability for clusters started on the wall. For example, at the ordinary transition, we would have

$$
P_{1}(t \rightarrow \infty, \Delta) \sim|\Delta|^{\beta_{1, \text { seed }}^{\mathrm{O}}, \quad \Delta<0 .}
$$

However, the surface exponents here show a similar pattern to their bulk counterparts and fulfill $\beta_{1, \text { seed }}^{\mathrm{O}}=\beta_{1 \text {,dens }}^{\mathrm{O}}=\beta_{1}^{\mathrm{O}}$, as can be shown by a field-theoretic derivation of an appropriate correlation function [14]. This kind of equality should also hold for the $\beta_{1}$ exponents at the special and surface transitions.

Numerically, the exponents at the ordinary transition have been measured very accurately using series expansions (for $d=1$ ) $[18,19]$, and Monte Carlo simulations (for $d=1$ and 2) [14-16]. However, there has been no numerical work to date on any of the other possible transitions on the boundary.

\section{A. Mean field theory}

Although a considerable amount of work has already been performed on surface DP [13-15,18], a comprehensive mean field analysis has been lacking. The purpose of this section is to provide such an analysis, and in the process we will derive several interesting results. The equation describing mean field DP with a surface is

$$
\partial_{t} n=D \nabla^{2} n-\Delta n-\lambda n^{2},
$$

with the boundary condition

$$
\left.D \partial_{x_{\perp}} n\right|_{x_{\perp}=0}=\left.\Delta_{s} n\right|_{x_{\perp}=0} .
$$

Here the variable $\Delta=\mu-\sigma$ is the difference between the rates for the $A \rightarrow \varnothing$ and $A \rightarrow A+A$ processes. Similarly we have the surface variable $\Delta_{s}$, and the bulk quadratic term is due to the reaction $A+A \rightarrow A$. Note that a surface $A+A$ $\rightarrow A$ reaction does not have to be included, as it is an irrelevant process in the renormalization group (RG) sense [13]. From the above equation (10), the bulk mean field exponents can easily be computed: $\nu_{\|}=1, \nu_{\perp}=1 / 2$, and $\beta=1$. Furthermore, with the inclusion of a boundary, we see that the correlation length exponents are unchanged at the wall but the surface $\beta_{1}$ exponents are altered. If we are interested in the mean field steady state, then we can replace Eq. (10) with

$$
D n^{\prime \prime}-\Delta n-\lambda n^{2}=0
$$

where $n^{\prime \prime} \equiv d^{2} n / d x_{\perp}^{2}$. The appropriate boundary condition (11) is given by

$$
D n_{s}^{\prime}=\Delta_{s} n_{s},
$$

where $n_{s}=\left.n\right|_{x_{\perp}=0}$, and $n_{s}^{\prime}=d n /\left.d x_{\perp}\right|_{x_{\perp}=0}$. Multiplying Eq. (12) by $n^{\prime}$ and integrating, we have

$$
\frac{1}{2} D n^{\prime 2}-\frac{1}{2} \Delta n^{2}-\frac{1}{3} \lambda n^{3}+C=0,
$$

where $C$ is a constant of integration. Using the bulk results $n^{\prime}=0$, and $n=(-\Delta) / \lambda$ for $\Delta<0$, or $n=0$ for $\Delta>0$, we have

$$
\begin{gathered}
\frac{\Delta_{s} n_{s}}{D}=-\left[\frac{\lambda}{D}\right]^{1 / 2}\left(n_{s}-\frac{|\Delta|}{\lambda}\right)\left(\frac{2}{3} n_{s}+\frac{|\Delta|}{3 \lambda}\right)^{1 / 2} \quad[\Delta<0], \\
\frac{\Delta_{s} n_{s}}{D}=-\left[\frac{\lambda}{D}\right]^{1 / 2} n_{s}\left(\frac{2}{3} n_{s}+\frac{\Delta}{\lambda}\right)^{1 / 2}[\Delta>0],
\end{gathered}
$$

where we have also used the boundary condition (13).

Ordinary transition: Consider the case where $\Delta_{s}>0$ and $\Delta \rightarrow 0^{-}$. In that case we expect $n=|\Delta| / \lambda \gg n_{s}$, and thus Eq. (15) yields $n_{s} \propto|\Delta|^{3 / 2}$, giving $\beta_{1}^{\mathrm{O}}=3 / 2$.

This exponent can also be derived on physical grounds as follows (see also Ref. [29]). At the ordinary transition the density falls to zero not exactly at the wall, but would rather reach zero a distance $l$ on the far side of the surface (if the density were appropriately continued). Hence the density on the boundary can be computed from $l n_{s}^{\prime}$. Since $l$ is a microscopic distance which remains finite even at the bulk critical point, one can compute the scaling of the surface density simply from $n_{s}^{\prime}=d n /\left.d x_{\perp}\right|_{x_{\perp}=0}$. Thus, from dimensional analysis, we see that $\beta_{1}^{\mathrm{O}}=3 / 2$.

Special transition: In this case if $\Delta_{s}=0$ we see from Eq. (15) that $n_{s}$ scales in the same way as the bulk density $n$, i.e., $\beta_{1}^{\mathrm{Sp}}=1$ [30]. Furthermore, a simple rewriting of Eqs. (15) and (16) reveals the scaling $\Delta_{s} \sim \Delta^{1 / 2}$, fixing the crossover exponent as $\phi_{1}=1 / 2$.

Surface transition: For this case $\Delta_{s}<0$ and $\Delta>0$, and, hence, from Eq. (16) we find $n_{s}=(3 / 2 D \lambda)\left[\Delta_{s}^{2}-D \Delta\right]$ for 0 $<D \Delta<\Delta_{s}^{2}$, and $n_{s}=0$ for $D \Delta>\Delta_{s}^{2}$. Hence the line in parameter space where the mean field surface transition occurs is given by $\Delta_{s}^{2}=D \Delta_{\text {critical }}$, and we then have $\beta_{1}^{\mathrm{S}}=1$. Note that this is the same value as in the bulk, a standard feature of the surface transition which is believed always to be in the same universality class as a $(d-1)$-dimensional bulk transition. As we are dealing with mean field theory this will of course yield the same exponent for the surface transition as in the bulk.

Extraordinary transition: In this case the surface density is of course nonzero both above and below the transition. However, if we expand $n_{s}$ in powers of $\Delta$ for $\Delta_{s}<0$ and $\Delta \rightarrow 0^{+}$or $0^{-}$, we see that these two expansions differ at third order [31], i.e., $n_{s}$ has a discontinuity in its third derivative at $\Delta=0$. Hence we identify $\beta_{1}^{\mathrm{E}}=3$. To the best of our knowledge, this transition does not seem to have been previously discussed in the literature. 
However, as is the case in equilibrium critical phenomena, we expect the extraordinary transition to be more general than the scenario described above. In fact, the extraordinary transition is associated with the onset of order in the bulk regardless of how the surface is ordered. In particular, for arbitrary values of $\Delta_{s}$, the surface can be ordered by applying the equivalent of a surface external magnetic field. For the BARW process this is simply the surface spontaneous particle creation reaction $\varnothing \rightarrow A$. Extending our previous mean field analysis to cover this case (sometimes called the normal transition), we recover precisely the same results as obtained above, with $\beta_{1}^{\mathrm{E}}=3$. Hence the important point for the extraordinary transition (as described in Ref. [32]) is that the surface must be active at $\Delta=0$ - the means by which this is achieved is unimportant.

Next, we consider the case where the bulk is exactly critical, i.e., $\Delta$ is exactly zero, and therefore the correlation lengths $\xi_{\|}$and $\xi_{\perp}$ diverge. In that the case the density in the bulk decays away as $d n / d t=-\lambda n^{2} \Rightarrow n \sim t^{-1}$. Hence, for the surface, we need now to include time dependence in our analysis, and therefore Eq. (12) is replaced by

$$
\dot{n}=D n^{\prime \prime}-\lambda n^{2}
$$

where $\dot{n}=\partial_{t} n$ and the boundary condition remains as given in Eq. (13). Multiplying both sides of Eq. (17) by $n^{\prime}$ and integrating, we obtain

$$
\int_{0}^{\infty} d x_{\perp} \dot{n} n^{\prime}=-\frac{1}{2 D} \Delta_{s}^{2} n_{s}^{2}-\frac{1}{3} \lambda\left(n^{3}-n_{s}^{3}\right),
$$

where we have used the conditions $n^{\prime}=0$ (in the bulk) and the boundary condition (13).

Exactly at the extraordinary transition: Here the density close to the wall will be in an active steady state, and hence nearby $\dot{n}$ will be close to zero. However, well away from the surface we expect to recover bulk behavior where $n^{\prime} \approx 0$ and $n \sim t^{-1}$. Hence, to leading order the integral on the left-hand side of Eq. (18) will be zero. Therefore, from Eq. (18), we find a steady state on the surface with $n_{s} \approx 3 \Delta_{s}^{2} / 2 D \lambda$. Furthermore, we expect that this active region will extend into the bulk, with the density decaying away asymptotically as $x_{\perp}^{-2}$. However, assuming the system is started with initial conditions at $t=0$ of constant density everywhere, then after a time $t$ this region will only extend into the bulk as far as $x_{\perp} \sim t^{1 / 2}$, where we will find a crossover to the bulk $t^{-1}$ density decay.

Exactly at the special transition: Here, where $\Delta_{s}=0$, we see that the mean field equation (18) is solved by $n_{s}=n$ $\sim t^{-1}$. Hence the surface density scales in the same way as in the bulk (see also Ref. [33]).

Exactly at the ordinary transition: Once again, if we start with initial conditions of uniform density at $t=0$, then at later times a depletion zone will be formed close to the surface. This zone will again extend a distance of order $t^{1 / 2}$ into the bulk. The surface scaling can now most simply be derived via dimensional analysis of the surface operator $\left.\partial_{x_{\perp}} n\right|_{x_{\perp}=0}$, yielding $n_{s} \sim t^{-3 / 2}$.

\section{B. Beyond mean field theory}

We expect that the phase diagram shown in Fig. 3 is generally valid for surface DP close to the upper critical dimension $d_{c}=4$. However, in $1+1$ dimensions, where the surface is just a zero-dimensional point, the phase diagram may look rather different. For example, for an inactive bulk, net particle production is only possible at one point. Furthermore, since particles will be constantly lost into the bulk, where they will decay away exponentially quickly, it will probably not be possible to form an active surface state (at least for finite particle production rates). If this is the case only the ordinary transition will be accessible in $d=1$. Furthermore, for arbitrary dimension, we note that a system which is simply cut off at $x_{\perp}=0$ can also only undergo an ordinary transition. This is a result of there being the same microscopic reaction rates on the surface as in the bulk. In low dimensions it becomes more and more difficult to induce an active state (since the fluctuations become larger), and, hence, if the bulk is adjusted to be at criticality, it follows that the surface (considered independently) would be inactive. Therefore, for the case of DP, one will only be able to find the ordinary transition (as was certainly the case in the simulations of Ref. [14]).

The scaling forms for the survival probability (at the ordinary transition) and correlation functions (at the special and ordinary transitions) were discussed in Refs. [13,14]. For example, at the ordinary transition, the survival probability for a cluster started on the wall at $t=0$ has the scaling form [14]

$$
P_{1}(t, \Delta)=|\Delta|^{\beta_{1}^{O}} \varphi_{1}\left(t / \xi_{\|}\right)
$$

Hence the average lifetime of finite clusters at the ordinary

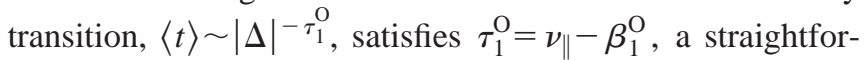
ward generalization of the bulk result. Previous series expansions in $1+1$ dimensions have indicated a value for $\tau_{1}^{\mathrm{O}}$ equal to unity [18], although very recent (and even more accurate) series results [19] have cast some doubt on this conclusion. No theoretical explanation for why $\tau_{1}^{\mathrm{O}}$ should be equal to unity has emerged.

The bulk density $n(\mathbf{x}, t)$ for a cluster initiated on the wall at $t=0$ is given by $[13,14]$

$$
\begin{gathered}
n_{1}^{\mathrm{O}}(x, t, \Delta)=|\Delta|^{\beta_{1}^{\mathrm{O}}+\beta} f_{1}\left[x / \xi_{\perp}, t / \xi_{\|}\right] \\
n_{1}^{\mathrm{Sp}}\left(x, t, \Delta, \Delta_{s}\right)=|\Delta|^{\beta_{1}^{\mathrm{Sp}}+\beta} \widetilde{f}_{1}\left[x / \xi_{\perp}, t / \xi_{\|}, \Delta_{s} /|\Delta|^{\phi_{1}}\right],
\end{gathered}
$$

where the surface exponents have been calculated to $O(\epsilon$ $=4-d$ ) in Ref. [13], giving $\beta_{1}^{\mathrm{O}}=3 / 2-7 \epsilon / 48, \beta_{1}^{\mathrm{Sp}}=1-\epsilon / 4$, and $\phi_{1}=1 / 2-\epsilon / 16$. The first of the expressions in Eq. (20) refers to the ordinary transition and the second to the special transition. Crudely speaking, the $\Delta$ prefactor in Eq. (20) comes from Eq. (19) for the probability that an infinite cluster can be grown from the seed, and from Eq. (4) for the conditional probability that the point $(\mathbf{x}, t)$ belongs to this cluster. At the ordinary transition, for example, it is then straightforward to derive hyperscaling relations for the mass of finite clusters which are seeded on the wall. This mass scales as $\left\langle s_{1}\right\rangle \sim|\Delta|^{-\gamma_{1}^{\mathrm{O}}}$, and, as shown in Ref. [14], one finds 


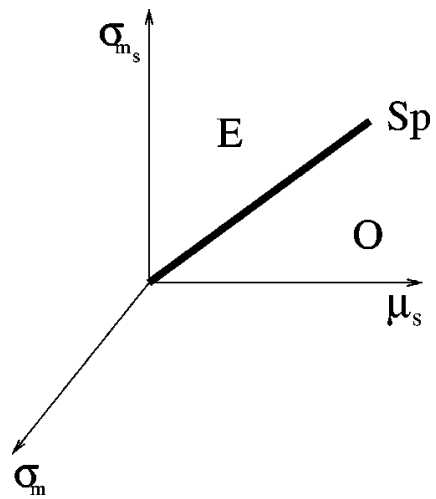

FIG. 4. Schematic mean field surface phase diagram for BARW. See the text for an explanation of the labeling.

$$
\nu_{\|}+d \nu_{\perp}=\beta_{1}^{\mathrm{O}}+\beta+\gamma_{1}^{\mathrm{O}}
$$

For a more detailed discussion of the DP ordinary transition, including other types of hyperscaling, we refer to Ref. [14].

\section{SURFACE BARW}

We now turn our attention to the main object of this paper, an understanding of the surface critical properties of BARW. We will begin by discussing the BARW surface phase diagram in various dimensions. The basic idea is that on the surface we may include not only the usual branching and annihilation reactions, but potentially also a parity symmetry breaking $A \rightarrow \varnothing$ reaction. Depending on whether or not the $A \rightarrow \varnothing$ reaction is actually present, we may then expect different surface universality classes according to whether the symmetry of the bulk is broken or respected at the surface. A similar situation in an equilibrium system was recently analyzed in Ref. [34]. We will find that the competition between the parity breaking $A \rightarrow \varnothing$ reaction and the BARW processes gives rise to some interesting phase diagrams.

\section{A. Mean field phase diagram}

The surface phase diagram for the mean field theory of BARW (valid for $d>d_{c}=2$ ) is shown in Fig. 4. Here $\sigma_{m}$ and $\sigma_{m_{s}}$ are the rates for the branching processes $A \rightarrow(m$ $+1) A$ in the bulk and at the surface, respectively, and $\mu_{s}$ is the rate for the surface spontaneous annihilation reaction $A$ $\rightarrow \varnothing$. Otherwise, the labeling is the same as that for the DP phase diagram (see Fig. 3).

The first feature to note is that the bulk is either active $\left(\sigma_{m}>0\right)$ or critical $\left(\sigma_{m}=0\right)$, but never inactive. Hence, unlike DP, there is no possibility of finding a surface transition, where the surface is critical with the bulk inactive. For the case where $\sigma_{m}=\mu_{s}=0$, we expect that for any finite value of the surface branching, the surface will become active. This corresponds to the extraordinary transition with an active surface and critical bulk. On the other hand, for $\sigma_{m}=\sigma_{m}$ $=0$ and $\mu_{s}>0$, the density at an (isolated) surface would decay away exponentially quickly due to the $A \rightarrow \varnothing$ reaction. Hence the bulk is critical, with the surface inactive; i.e., the ordinary transition. Consequently with $\sigma_{m}=0$, but both $\mu_{s}$ and $\sigma_{m_{s}}$ nonzero, there should be a line of special transitions (a)

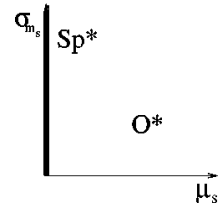

(b)

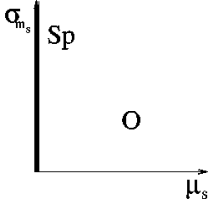

FIG. 5. Schematic surface phase diagrams for BARW in $d=1$ for (a) $\sigma_{m}<\sigma_{m \text {,critical }}$, and (b) $\sigma_{m}=\sigma_{m \text {,critical }}$. See the text for an explanation of the labeling.

dividing the extraordinary and ordinary regions. This explains the general features of the phase diagram in Fig. 4.

At a more quantitative level, the mean field equation for BARW is very similar to that for DP,

$$
\partial_{t} n=D \nabla^{2} n-\Delta n-\lambda n^{2},
$$

with the boundary condition

$$
\left.D \partial_{x_{\perp}} n\right|_{x_{\perp}=0}=\left.\Delta_{s} n\right|_{x_{\perp}=0} .
$$

However, the values of the $\Delta, \Delta_{s}$ parameters are now different: $\Delta=-m \sigma_{m}$ and $\Delta_{s}=-m \sigma_{m_{s}}+\mu_{s}$. The fact that $\Delta$ is always nonpositive excludes any possibility of a surface transition. Otherwise we expect the same mean field exponents as in DP for the special and ordinary transitions [30] (except for the $\beta_{1 \text {,seed }}$ exponents; see below). However, the nonpositivity of $\Delta$ also leads to an ambiguity associated with the definition of $\beta_{1 \text {,dens }}^{\mathrm{E}}$ : we would have to know the behavior of the surface density on both sides of the extraordinary transition if we wanted to isolate the discontinuity and extract the exponent.

We can also consider the mean field behavior of the $\beta_{1 \text {,seed }}$ exponents, which is very different from the corresponding behavior in DP. Consider placing two particles next to the surface at $t=0$. From the recurrence properties of random walks we see that, regardless of the reaction rates on the surface or in the bulk, there is a finite chance that the two particles will never meet again. Hence the survival probability is nonzero, and thus $\beta_{1 \text {,seed }}=0$ in mean field theory for the ordinary and special transitions.

\section{B. Phase diagram in $1+1$ dimensions}

Next we turn our attention to the phase diagram for $1+1$ dimensions shown in Fig. 5. Although we will make a few remarks below, we will postpone a proper justification until we have discussed the appropriate field theory in Sec. IV D. The phase diagram looks quite different from its mean field analog due in part to the shift of the bulk critical point away from zero branching rate, but also due to the absence of any extraordinary transition (for finite reaction rates). Physically, this is due to the fact that excess particle production (with a finite reaction rate) at a zero-dimensional surface is simply not efficient enough to generate an active state, due to leakage into the critical bulk (which for $\sigma_{m}<\sigma_{m \text {,critical }}$ is controlled by the fixed point of the $A+A \rightarrow \varnothing$ reaction [6]).

However, for infinite branching rates and/or if the reaction $\varnothing \rightarrow A$ is added at the surface, then an extraordinary transition should become accessible, although we will not consider this case in any further detail. The other main fea- 
tures of the phase diagram in $1+1$ dimensions are outlined below.

$S p^{*}$ : For $\sigma_{m}<\sigma_{m \text {, critical }}$ and $\mu_{s}=0$, the system is controlled everywhere by the annihilation fixed point. In that case one has the special transition, but now in a slightly different sense to what we have seen before. In this region it is not possible to obtain an active state either on the surface or in the bulk, by small changes in the bulk and/or surface branching rates. Hence this "transition" is actually entirely controlled by the $A+A \rightarrow \varnothing$ process with the branching playing essentially no role. Thus we have marked this "transition" as Sp* in Fig. 5(a). This simpler and analytically tractable case was already extensively analyzed in Ref. [33]. We will postpone further theoretical discussion until Sec. IV D 4.

$S p$ : Next we consider the special transition at $\sigma_{m}$ $=\sigma_{m \text {, critical }}, \mu_{s}=0$. This transition borders the bulk active phase, and hence will belong to a quite different universality class to that described immediately above, and will instead be similar to the special transitions discussed in earlier sections [although fluctuations will now be very important for this $(1+1)$-dimensional case].

$O^{*}$ : For $\mu_{s}>0$ and $\sigma_{m}<\sigma_{m \text {, critical }}$, the presence of the $A \rightarrow \varnothing$ reaction on the surface gives rise to an ordinary "transition." However, as explained above, the branching process again plays essentially no role here. Further details of this O* "transition" are provided in Sec. IV D 4.

$O$ : Finally, at $\sigma_{m}=\sigma_{m \text {, critical }}, \mu_{s}>0$, we expect an ordinary transition similar in character to the ordinary transitions discussed in previous sections [although in this $(1+1)$ dimensional case the fluctuations are again very important].

\section{Scaling theory}

In this section we construct a scaling theory for the survival probabilities and correlation functions at the $\mathrm{Sp}$ and $\mathrm{O}$ transitions. This scaling theory is certainly valid for the mean field regime but there are, however, subtleties involved in its application to the fluctuation dominated regime for $d \leqslant 2$. The extent of its validity in that region will be discussed in detail in Sec. IV D. When writing down this scaling theory we must also bear in mind the important distinction between the $\beta_{1 \text {,dens }}$ and $\beta_{1 \text {,seed }}$ exponents. We begin by giving a scaling form for the survival probability $P_{1}(t, \Delta)$, where $\Delta$ is the deviation from bulk BARW criticality. For example, at the ordinary transition, for a seed placed on the wall at $\mathbf{x}=\mathbf{0}$ and $t=0$, we have

$$
P_{1}(t, \Delta)=|\Delta|^{\beta_{1, \text { seed }}^{\mathrm{O}} \Phi_{1}\left(t / \xi_{\|}\right)}
$$

It is then straightforward to compute the average lifetime of finite clusters, $\langle t\rangle \sim|\Delta|^{-\tau_{1}}$, where $\tau_{1}^{\mathrm{O}}=\nu_{\|}-\beta_{1 \text {,seed }}^{\mathrm{O}}$, just as in the case of DP.

Next, we consider the coarse-grained particle density $n_{1}$ at the point $(\mathbf{x}, t)$ for a cluster grown from a seed located next to the wall at $\mathbf{x}=\mathbf{0}$ and $t=0$. At the ordinary transition we have

$$
n_{1}(x, t, \Delta)=|\Delta|^{\beta_{1, \text { seed }}^{\mathrm{O}}+\beta_{\text {dens }} g_{1}\left(x / \xi_{\perp}, t / \xi_{\|}\right) .}
$$

As was the case for DP, the $\Delta$ prefactor in Eq. (25) comes from Eq. (24) for the probability that an infinite cluster can be grown from the seed, and from Eq. (4) for the (conditional) probability that the point $(\mathbf{x}, t)$ belongs to this cluster. The shape of the cluster is governed by the scaling function $g_{1}$ and we assume that the density is measured at a finite angle away from the wall. If the density is measured along the wall, we have, instead,

$$
n_{11}(x, t, \Delta)=|\Delta|^{\beta_{1, \text { seed }}^{\mathrm{O}}+\beta_{1, \text { dens }}^{\mathrm{O}}{ }_{11}\left(x / \xi_{\perp}, t / \xi_{\|}\right)},
$$

as we pick up a factor $|\Delta|^{\beta_{1, \text { dens }}^{O}}$ rather than $|\Delta|^{\beta_{\text {dens }}}$ from the probability that $(\mathbf{x}, t)$ at the wall belongs to the cluster.

The above correlation functions need only be modified slightly to be valid at the special transition. If $\Delta_{s}$ (the deviation of the surface from criticality) is a relevant parameter, then we must take care to include the extra variable $\Delta_{s} /|\Delta|^{\phi_{1}}$ in the scaling function. The scaling form replacing Eq. (25) then becomes

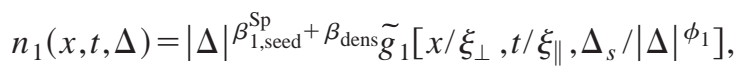

where $\phi_{1}$ is a crossover exponent associated with the multicritical special transition. Similarly, Eq. (26) is replaced by

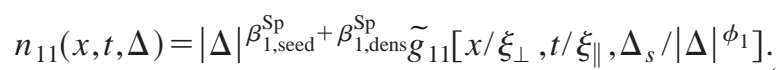

Note, however, that there are subtleties concerning the special transition in $1+1$ dimensions which will be discussed in Sec. IV D 1.

At the ordinary transition, for example, we can use the above scaling forms to derive some further exponent equalities. The average size of finite clusters

$$
\left\langle s_{1}\right\rangle \sim|\Delta|^{-\gamma_{1}^{\mathrm{O}}}
$$

follows from integrating the cluster density (25) over space and time, where the surface (susceptibility) exponent $\gamma_{1}^{\mathrm{O}}$ is related to the previously defined exponents via

$$
\nu_{\|}+d \nu_{\perp}=\beta_{1, \text { seed }}^{\mathrm{O}}+\beta_{\mathrm{dens}}+\gamma_{1}^{\mathrm{O}} .
$$

Analogously, by integrating the cluster wall density (26) over the $(d-1)$-dimensional wall and time, we obtain the average size of finite clusters on the wall,

$$
\left\langle s_{1,1}\right\rangle \sim|\Delta|^{-\gamma_{1,1}^{\mathrm{O}}}
$$

where

$$
\nu_{\|}+(d-1) \nu_{\perp}=\beta_{1, \text { seed }}^{\mathrm{O}}+\beta_{1, \text { dens }}^{\mathrm{O}}+\gamma_{1,1}^{\mathrm{O}} .
$$

Note that if the $\gamma$ susceptibility exponents obtained from Eqs. (30) and (32) are negative, then they should be replaced by zero in Eqs. (29) and (31).

\section{Field theory}

In order to properly understand the effects of fluctuations, and to justify some of the scaling forms proposed in the last section, we now turn to the development of a field theory for surface BARW. We will begin by reviewing the field theory for BARW in the bulk, before moving on to derive the ap- 
propriate surface actions. The bulk BARW action, written in terms of the response field $\hat{\psi}(\mathbf{x}, t)$ and the "density" field $\psi(\mathbf{x}, t)$, is given by [6]

$$
\begin{aligned}
S_{\text {bulk }}^{\text {bare }}[\psi, \hat{\psi} ; \tau]= & \int d^{d} x\left[\int _ { 0 } ^ { \tau } d t \left[\hat{\psi}(\mathbf{x}, t)\left[\partial_{t}-D \nabla^{2}\right] \psi(\mathbf{x}, t)\right.\right. \\
& -\lambda\left[1-\hat{\psi}(\mathbf{x}, t)^{2}\right] \psi(\mathbf{x}, t)^{2} \\
& \left.+\sigma_{m}\left[1-\hat{\psi}(\mathbf{x}, t)^{m}\right] \hat{\psi}(\mathbf{x}, t) \psi(\mathbf{x}, t)\right] \\
& \left.-\psi(\mathbf{x}, \tau)-n_{0} \hat{\psi}(\mathbf{x}, 0)\right] .
\end{aligned}
$$

Here the terms on the first line of Eq. (33) represent diffusion of the particles (with continuum diffusion constant $D$ ). The second line describes the annihilation reaction (with continuum rate $\lambda$ ), while the terms on the third line represent the branching process (with continuum rate $\sigma_{m}$ ). The final two terms represent, respectively, a contribution due to the projection state (see Ref. [35]), and the initial condition (an uncorrelated Poisson distribution with mean $n_{0}$ ). In the following we will restrict ourselves to the case of even $m$, since it is known that the odd $m$ case belongs to the DP universality class [6].

The action given in Eq. (33) is a bare action. In order to properly include fluctuation effects, one must be careful to include processes generated by a combination of branching and annihilation. In other words, in addition to the process $A \rightarrow(m+1) A$, the reactions $A \rightarrow(m-1) A, \ldots, A \rightarrow 3 A$ need to be included. These considerations lead to the full action

$$
\begin{aligned}
S_{\text {bulk }}[\psi, \hat{\psi} ; \tau]= & \int d^{d} x\left[\int _ { 0 } ^ { \tau } d t \left(\hat{\psi}(\mathbf{x}, t)\left[\partial_{t}-D \nabla^{2}\right] \psi(\mathbf{x}, t)\right.\right. \\
& +\sum_{l=1}^{m / 2} \sigma_{2 l}\left[1-\hat{\psi}(\mathbf{x}, t)^{2 l}\right] \hat{\psi}(\mathbf{x}, t) \psi(\mathbf{x}, t) \\
& \left.-\lambda\left[1-\hat{\psi}(\mathbf{x}, t)^{2}\right] \psi(\mathbf{x}, t)^{2}\right) \\
& \left.-\psi(\mathbf{x}, \tau)-n_{0} \hat{\psi}(\mathbf{x}, 0)\right] .
\end{aligned}
$$

Notice also that (for even $m$ ) the action (34) is invariant under the "parity" transformation

$$
\hat{\psi}(\mathbf{x}, t) \rightarrow-\hat{\psi}(\mathbf{x}, t), \quad \psi(\mathbf{x}, t) \rightarrow-\psi(\mathbf{x}, t) .
$$

This symmetry corresponds physically to particle conservation modulo 2 . The presence of this extra symmetry now takes the system away from the DP universality class, and into a new class: that of branching-annihilating random walks with an even number of offspring.

Close to the upper critical dimension $d_{c}=2$, the renormalization of the above action is quite straightforward (here we quote the results from Ref. [6]). Only the branching and annihilation rates need be renormalized, and in particular there are no diffusion constant or field renormalizations. Furthermore, if we are close to the annihilation fixed point, then the RG eigenvalue of the branching parameter becomes $y_{\sigma_{m}}$ $=2-m(m+1) \epsilon / 2+O\left(\epsilon^{2}\right)$, where $\epsilon=2-d$. Hence we see that the lowest branching process is actually the most relevant. Therefore, close to two dimensions, where the branching remains relevant, we expect to find an active state even for very small values of the branching (in agreement with the mean field phase diagram). Furthermore, the fact that there is only one eigenvalue to compute perturbatively (as the renormalization of the annihilation rate can be performed to all orders [36]), means that there is only one independent exponent. Hence, close to two dimensions, the order parameter exponent can be shown to be $\beta_{\text {dens }}=d \nu_{\perp}$.

However, inspection of the above RG eigenvalue $y_{\sigma_{m}}$ shows that it eventually becomes negative (if the one loop result is to be believed). In that case we expect a major change in the behavior of the system, since the branching process will no longer be relevant at the annihilation fixed point. The critical transition point is then shifted with the active state only being present for values of the branching greater than some positive critical value [as indicated in Fig. 2(b)]. Consequently, we see that there is a second critical dimension $d_{c}^{\prime} \approx 4 / 3$ whose presence immediately rules out any possibility of accessing the nontrivial behavior expected in $d=1$ via perturbative epsilon expansions down from $d$ $=2$. Instead cruder techniques (such as the loop expansion in fixed dimension) must be employed [6]. We now turn to the derivation of the surface actions appropriate for the cases $\mu_{s}=0$ and $\mu_{s} \neq 0$.

\section{1. $\mu_{s}=0$ field theory}

Starting from an appropriate master equation for the system on a lattice, the form of the surface action can be derived using standard techniques [35,36]. After mapping to the continuum theory, we find the bare action $S^{\text {bare }}=S_{\text {bulk }}^{\text {bare }}+S_{1}^{\text {bare }}$, with $S_{\text {bulk }}$ given by Eq. (33), and

$$
S_{1}^{\mathrm{bare}}=\int d^{d-1} x_{\|} \int_{0}^{\tau} d t\left(\sigma_{m_{s}}\left[1-\hat{\psi}_{s}^{m}\right] \hat{\psi}_{s} \psi_{s}\right),
$$

where $\hat{\psi}_{s}=\hat{\psi}\left(\mathbf{x}_{\|}, x_{\perp}=0, t\right)$ and $\psi_{s}=\psi\left(\mathbf{x}_{\|}, x_{\perp}=0, t\right)$. Note that the terms representing the annihilation reaction $A+A \rightarrow \varnothing$ are irrelevant on the surface close to the upper critical dimension.. The classical field equations for the above action can be derived by taking the variational derivatives of the action $S^{\text {bare }}=S_{\text {bulk }}^{\text {bare }}+S_{1}^{\text {bare }}$ with respect to the fields $\hat{\psi}$ and $\psi$. These equations are solved by $\hat{\psi}=\hat{\psi}_{s}=1$, with $\psi$ satisfying

$$
\partial_{t} \psi=D \nabla^{2} \psi-\Delta \psi-2 \lambda \psi^{2}
$$

where $\Delta=-m \sigma_{m}$, and with the boundary condition $\left.D \partial_{x_{\perp}} \psi\right|_{x_{\perp}=0}=\Delta_{s} \psi_{s}$, where $\Delta_{s}=-m \sigma_{m_{s}}$. These mean field results are in agreement with our analysis in Sec. IV A. Furthermore, we note that a boundary term of the form $\left.\hat{\psi}_{s} \partial_{x_{\perp}} \psi\right|_{x_{\perp}=0}$, although marginal from power counting arguments, is actually always redundant (even in the regime where mean field theory no longer applies). This is also the case for the surface action in DP (see Ref. [13]).

However, if we are properly to include fluctuation effects, we must again take care to include surface terms generated 
by a combination of branching and annihilation (as in the bulk). This leads to the full surface action

$$
S_{1}=\int d^{d-1} x_{\|} \int_{0}^{\tau} d t\left[\sum_{l=1}^{m / 2} \sigma_{2 l_{s}}\left[1-\hat{\psi}_{s}^{2 l}\right] \hat{\psi}_{s} \psi_{s}\right] .
$$

Note also that the parity symmetry (35) is preserved for the $\mu_{s}=0$ model at the wall, as well as in the bulk.

Power counting on the above action reveals that the surface branching rates $\sigma_{2 l_{s}}$ all have naive dimension [ $\left.\sigma_{2 l_{s}}\right]$ $\sim \kappa^{1}$, where $\kappa$ denotes an inverse length scale. However, below two dimensions this scaling dimension will be renormalized downwards (this can be seen physically as a result of processes like $A \rightarrow 3 A \rightarrow A$ rendering the branching process less efficient). As a result of this renormalization, we expect the lowest generated process [i.e., with $l=1$ in Eq. (38)] will become the most relevant (as it was in the bulk). Nevertheless, despite this downward renormalization, close enough to two dimensions, the scaling dimension of the most relevant coupling $\sigma_{2}$ will remain positive, and thus under the RG will flow to $\infty$ for all nonzero starting values. This state of affairs corresponds to the extraordinary transition where the surface is active while the bulk is critical. On the other hand, at bulk criticality and with $\sigma_{2_{s}}=0$, we have a multicritical special transition point. In this case, after writing down and solving the appropriate RG equations (exactly along the lines of Refs. $[6,13]$ ), one can derive the scaling results for the density quoted in Sec. IV C, where we can see that the independent renormalization of $\sigma_{2}$ contributes to the crossover exponent $\phi_{1}$. Furthermore, since there is no field renormalization (either at the surface or in the bulk), this implies that the exponent $\beta_{1, \text { dens }}^{\mathrm{Sp}}$ is just the same as in the bulk, i.e., $\beta_{1, \mathrm{dens}}^{\mathrm{Sp}}=\beta_{\mathrm{dens}}$. However, we must again stress that this result is only true close to $d=2$.

The situation in $d=1$ is rather different, partly due to the shift of the bulk critical point away from $\sigma_{m}=0$. This means that the $d=1$ transition at $\sigma_{m}=\sigma_{m \text {, critical }}$ cannot be based on perturbative epsilon expansion calculations down from two dimensions. However, we can say a little more if we first consider the regime $\sigma_{m}<\sigma_{m \text {,critical }}$ in $d=1$, where the bulk is controlled solely by the $A+A \rightarrow \varnothing$ reaction. In that case we expect the scaling dimension of all the $\sigma_{2 l_{s}}$ to be negative in $d=1$, following the downward trend in the renormalization mentioned above. In that case surface branching is then irrelevant in $d=1$, leading to the $\mathrm{Sp}^{*}$ special "transition." Similarly, at the $\mathrm{Sp}$ transition at $\sigma_{m}=\sigma_{m \text {, critical }}$, we might again expect $\sigma_{2 l_{s}}$ to be irrelevant. This will be reflected in the scaling functions for the density, where (unusually) the crossover term $\Delta_{s} /|\Delta|^{\phi_{1}}$ will now be absent. However, the surface exponents here will presumably be unrelated to the bulk exponents, since the absence of field renormalization mentioned above is not expected to hold all the way down to $d=1$.

Hence, if the above scenario is correct, we do not expect to see an extraordinary transition in $d=1$ for any finite value of the surface branching, since the surface branching will always be irrelevant. We have confirmed this analysis numerically: our simulations have found no evidence of an active surface state for $\sigma_{m} \leqslant \sigma_{m \text {, critical }}$ even for very high values of the surface branching parameter in a fermionic lattice model in $1+1$ dimensions (see Sec. VI for further details).

\section{2. $\boldsymbol{\mu}_{s} \neq \mathbf{0}$ field theory}

In this case the reaction $A \rightarrow \varnothing$ is now possible, but only at sites on the wall. In the bosonic field theory language employed above, we have the bare action $S^{\text {bare }}=S_{\text {bulk }}^{\text {bare }}$ $+S_{2}^{\text {bare }}$, where

$$
S_{2}^{\mathrm{bare}}=\int d^{d-1} x_{\|} \int_{0}^{\tau} d t\left(\sigma_{m_{s}}\left[1-\hat{\psi}_{s}^{m}\right] \hat{\psi}_{s} \psi_{s}+\mu_{s}\left[\hat{\psi}_{s}-1\right] \psi_{s}\right) .
$$

Symmetry (35) is now broken by the surface term proportional to $\mu_{s}$, which describes the $A \rightarrow \varnothing$ reaction. Repeating our derivation of the classical (mean field) equation for the $\psi$ density field, we find

$$
\partial_{t} \psi=D \nabla^{2} \psi-\Delta \psi-2 \lambda \psi^{2},
$$

where $\Delta=-m \sigma_{m}$, and with the boundary condition $\left.D \partial_{x_{\perp}} \psi\right|_{x_{\perp}=0}=\Delta_{s} \psi_{s}$, where $\Delta_{s}=-m \sigma_{m_{s}}+\mu_{s}$. This is in agreement with the mean field analysis given in Sec. IV A. Note that a boundary term of the form $\left.\hat{\psi}_{s} \partial_{x_{\perp}} \psi\right|_{x_{\perp}=0}$ is again always redundant.

Action (39) is a bare action whose terms simply represent the reactions $A \rightarrow(m+1) A$ and $A \rightarrow \varnothing$ at the surface. Clearly, however, from these two reactions we can generate the hierarchy of processes $A \rightarrow m A, A \rightarrow(m-1) A, \ldots, A$ $\rightarrow 2 A$. Hence we must replace the above bare surface action with

$S_{2}=\int d^{d-1} x_{\|} \int_{0}^{\tau} d t\left[\sum_{l=1}^{m} \sigma_{l_{s}}\left[1-\hat{\psi}_{s}^{l}\right] \hat{\psi}_{s} \psi_{s}+\mu_{s}\left[\hat{\psi}_{s}-1\right] \psi_{s}\right]$.

The renormalization of the action (41) is now somewhat different from the $\mu_{s}=0$ case. We again expect that we need only keep the lowest generated branching term on the surface, namely, that with $l=1$ in Eq. (41). As before, we expect fluctuations to lower the scaling dimension of this coupling from its mean field value (although actually in $d=2$ this suppression will only be logarithmic). On the other hand, the efficacy of the $A \rightarrow \varnothing$ reaction is certainly not reduced by fluctuations. Hence we expect that $\Delta_{s} \rightarrow \mu_{s}-\sigma_{1_{s}}$ will always run to the fixed point at $\infty$, corresponding to the ordinary transition. In that case the surface $\beta_{1, \text { dens }}^{\mathrm{O}}$ exponent is again simply related to the bulk result due to the absence of any surface field renormalization. This exponent can be computed from the scaling of the surface operator $\partial \psi /\left.\partial x_{\perp}\right|_{x_{\perp}=0}$, where the $x_{\perp}$ derivative simply brings out an extra factor of $\nu_{\perp}$ from the scaling function, giving $\beta_{1, \text { dens }}^{\mathrm{O}}=\beta_{\text {dens }}+\nu_{\perp}$. Using the result $\beta_{\mathrm{dens}}=d \nu_{\perp}$ from Ref. [6], we see that $\beta_{1 \text {,dens }}^{\mathrm{O}}$ $=(d+1) \nu_{\perp}$. Again we stress that this result is only true close to two dimensions. The more interesting transition at $\sigma_{m}=\sigma_{m, \text { critical }}$ in $d=1$ is not perturbatively accessible in epsilon expansions down from $d=2$. Nevertheless, we still expect the same general picture to hold with the surface branching always being irrelevant, leading to the $\mathrm{O}^{*}\left(\sigma_{m}\right.$ $\left.<\sigma_{m, \text { critical }}\right)$ or $\mathrm{O}\left(\sigma_{m}=\sigma_{m, \text { critical }}\right)$ transitions. 


\section{Discussion}

From the above analysis we can understand the structure of the phase diagram close to two dimensions. For $\mu_{s}=0$ this is similar to the mean field picture, with a special transition point at $\sigma_{m}=\sigma_{m_{s}}=0$, and with the extraordinary transition for $\sigma_{m}=0, \sigma_{m_{s}}>0$. On the other hand, for $\mu_{s}>0$, the picture is very different from mean field theory, with renormalization effects ensuring that only the ordinary transition is accessible. However, actually in $d=2$ this might be hard to observe, since in that case the surface branching is only marginally less relevant.

One would now like to use actions (36) and (39) as the starting point for a field-theoretic investigation of the $\sigma_{m}$ $=\sigma_{m \text {, critical }}$ transitions in $d=1$, where one would like to identify two independent, nontrivial surface $\beta_{1}$ exponents (a feature which is certainly indicated by our simulations; see Sec. VI). Surprisingly, our numerical results also indicate that these surface $\beta_{1}$ exponents "swap" if the $\mu_{s}=0$ and $\mu_{s}$ $\neq 0$ cases are interchanged (i.e., $\beta_{1, \text { dens }}^{\mathrm{O}}=\beta_{1 \text {,seed }}^{\mathrm{Sp}}$ and $\beta_{1 \text {,seed }}^{\mathrm{O}}$ $=\beta_{1 \text {,dens }}^{\mathrm{Sp}}$. These interesting results certainly merit further analysis. Unfortunately the use of field-theoretic techniques here will be plagued by precisely the same problems as afflicted the bulk calculation, namely, the appearance of a second critical dimension $d_{c}^{\prime}$. Hence one would be forced into using uncontrolled techniques (such as the truncated loop expansion in fixed dimension) whose values for the bulk exponents are known to be in rather poor agreement with numerics [6]. Furthermore, field and diffusion constant renormalizations, which will be of considerable importance in $d=1$, are not adequately taken into account in the truncated loop theory. In fact these renormalizations only appear at two loop order. Unfortunately the authors of Ref. [6] were unable to show that a meaningful truncated loop theory exists at all at the level of two loops. In addition, further technical difficulties exist for $d<d_{c}^{\prime}$ involving dangerous irrelevant variables, which have so far prevented a derivation of scaling relations at criticality even in the bulk. In the light of these problems we have not attempted to extend the truncated loop analysis to surface BARW.

\section{Results from $A+A \rightarrow \varnothing$}

The $(1+1)$-dimensional regime which should prove more amenable to field-theoretic analysis is when both the bulk and surface branching processes are unimportant, and hence we should be able to use results derived solely from the $A$ $+A \rightarrow \varnothing$ reaction. This is the case for the region $\sigma_{m}$ $<\sigma_{m \text {,critical }}$ in $d=1$ [see Fig. 5(a)]. The Sp* "transition" was fully analyzed in Ref. [33], which predicted a $t^{-1 / 2}$ decay both in the bulk and at the wall, with a density excess at the wall. We note that the critical $\mathrm{Sp}^{*}$ state in $d=1$ can be characterized in two ways: as a decaying density, or as a survival probability. If we place two particles close together in the bulk at $t=0$, then, from simple random walk theory, the probability these particles are still alive at time $t$ scales as $t^{-1 / 2}$. Hence, in the bulk, these two ways of characterizing this phase scale in the same way. However, if the two particles are released next to the wall, then it is easy to show (using the method of images) that the survival probability now decays as $t^{-1}[37,38]$. Therefore, these two characterizations of the $\mathrm{Sp}^{*}$ state do not scale in the same way close to the wall.

Since the bulk at the $d=1 \mathrm{O}^{*}$ transition is controlled solely by the reaction $A+A \rightarrow \varnothing$, its properties can also be inferred. The relevant surface operator is again just $\left.\partial_{x_{\perp}} \psi\right|_{x_{\perp}=0}$. Therefore, since distance still scales as $[x] \sim t^{1 / 2}$, we can obtain the required surface scaling from simple dimensional analysis. Since the bulk $d=1$ density decays as $t^{-1 / 2}$, we see that the surface density must decay as $t^{-1}$.

However, we must emphasize again at this point that the methods and results mentioned here are only applicable where both the surface and bulk branching processes are unimportant. Unfortunately, therefore, the more interesting Sp and $\mathrm{O}$ transitions in $1+1$ dimensions remain out of reach.

Hence, given the fundamental difficulties associated with the field theory, it seems fruitful to search for alternative approaches to the problem which might shed some further light on the interesting properties of the surface $\beta_{1}$ exponents in $d=1$. One such alternative is provided by the theory of quantum spin Hamiltonians, to which we turn in Sec. IV E.

\section{E. Exact results}

In this section we will derive some exact results for the surface $\beta_{1}$ exponents in $1+1$ dimensions at the $\mathrm{O}$ and $\mathrm{Sp}$ transitions. The methods are a straightforward extension of the work in Refs. [28,39]. The starting point is the following set of rules for BARW with $m=2$ in $1+1$ dimensions:

$$
\begin{gathered}
\varnothing A \leftrightarrow A \varnothing \quad \text { with rate } D / 2, \\
A A \rightarrow \varnothing \varnothing \text { with rate } \lambda,
\end{gathered}
$$

$$
\varnothing A \varnothing \leftrightarrow A A A \text { and } \varnothing A A \leftrightarrow A A \varnothing \text { with rate } \alpha / 2 \text {. }
$$

Note that these rules are fermionic in character (no more than one particle per site is permitted) in contrast to the bosonic rules employed in the derivation of the earlier field theory. The model described in Eq. (42) can be transformed into a spin picture by writing the configuration of a semi-infinite system as a vector $\left|s_{1}, s_{2}, s_{3}, \ldots\right\rangle$, where $s_{i}=1 / 2$ if the $i$ th site is empty, and $s_{i}=-1 / 2$ if that site is occupied. Hence the system ket is given by

$$
|P(t)\rangle=\sum_{\left\{s_{i}\right\}} P\left(\left\{s_{i}\right\} ; t\right)\left|\left\{s_{i}\right\}\right\rangle
$$

and the equation governing the time evolution is

$$
\partial_{t}|P(t)\rangle=-\mathcal{H}|P(t)\rangle
$$

where, using a representation in terms of Pauli matrices, and defining $n_{k}=\left(1-\sigma_{k}^{z}\right) / 2, \mathrm{v}_{k}=1-n_{k}, s_{k}^{ \pm}=\left(\sigma_{k}^{x} \pm i \sigma_{k}^{y}\right) / 2$, we have [28] 


$$
\begin{aligned}
\mathcal{H}= & \frac{1}{2} \sum_{k=1}^{\infty}\left(D\left[n_{k} \mathrm{v}_{k+1}+\mathrm{v}_{k} n_{k+1}-s_{k}^{+} s_{k+1}^{-}-s_{k}^{-} s_{k+1}^{+}\right]\right. \\
& \left.+2 \lambda\left[n_{k} n_{k+1}-s_{k}^{+} s_{k+1}^{+}\right]\right)+\frac{\alpha}{2} \sum_{k=2}^{\infty}\left(1-\sigma_{k-1}^{x} \sigma_{k+1}^{x}\right) n_{k} \\
= & D H^{\mathrm{SEP}}+\lambda H^{\mathrm{RSA}}+\alpha H^{\mathrm{BARW}} \\
= & D \sum_{k=1}^{\infty} h_{k}^{\mathrm{SEP}}+\lambda \sum_{k=1}^{\infty} h_{k}^{\mathrm{RSA}}+\alpha \sum_{k=2}^{\infty} h_{k}^{\mathrm{BARW}} .
\end{aligned}
$$

Here we have used some of the notation of Ref. [28], where symmetric exclusion process (SEP) refers to the diffusion piece, random-sequential adsorption (RSA) to the annihilation piece, and BARW to the branching piece of the "quantum Hamiltonian.' Notice that the boundary has been included in (45), since particles may not hop to the left of site 1 , and the annihilation/branching processes have also been restricted to sites $1,2,3 \ldots$ Hence, the above operator $\mathcal{H}$ governs the evolution of a $(1+1)$-dimensional BARW system without an $A \rightarrow \varnothing$ reaction at the boundary. Averages are calculated using the projection state \langle|$=\Sigma_{\left\{s_{i}\right\}}\left\langle\left\{s_{i}\right\}\right|$, i.e., $\langle\mathcal{F}\rangle=\langle|\mathcal{F}| P(t)\rangle$. Following Ref. [28], we now define an operator $\mathcal{D}$ where

$$
\mathcal{D}=\gamma_{-1} \gamma_{0} \gamma_{1} \gamma_{2} \ldots
$$

with

$$
\begin{gathered}
\gamma_{2 k-1}=\frac{1}{2}\left[(1+i) \sigma_{k}^{z}-(1-i)\right], \\
\gamma_{2 k}=\frac{1}{2}\left[(1+i) \sigma_{k}^{x} \sigma_{k+1}^{x}-(1-i)\right] .
\end{gathered}
$$

Defining a new "quantum Hamiltonian" as $\widetilde{\mathcal{H}}$ $=\left[\mathcal{D}^{-1} H \mathcal{D}\right]^{T}$, we find

$$
\begin{aligned}
\widetilde{\mathcal{H}}= & {[D-\lambda] \sum_{k=1}^{\infty} h_{k}^{\mathrm{BARW}}+[\alpha+\lambda] \sum_{k=1}^{\infty} h_{k}^{\mathrm{SEP}}+\lambda \sum_{k=1}^{\infty} h_{k}^{\mathrm{RSA}} } \\
& +\frac{\lambda}{2}\left[n_{1} n_{0}-s_{1}^{+} s_{0}^{+}+n_{1} \mathrm{v}_{0}-s_{1}^{+} s_{0}^{-}\right]
\end{aligned}
$$

where we have used the commutation rules described in detail in Ref. [28]. Hence, when $D=\lambda+\alpha$, we have the following processes occurring:

$$
\begin{gathered}
\varnothing_{i} A_{i+1} \leftrightarrow A_{i} \varnothing_{i+1} \text { rate }(\lambda+\alpha) / 2, \quad i=1,2,3, \ldots, \\
A_{i} A_{i+1} \rightarrow \varnothing_{i} \varnothing_{i+1} \text { rate } \lambda, \quad i=1,2,3, \ldots, \\
\varnothing_{i-1} A_{i} \varnothing_{i+1} \leftrightarrow A_{i-1} A_{i} A_{i+1} \text { rate } \alpha / 2, i=1,2,3, \ldots, \\
\varnothing_{i-1} A_{i} A_{i+1} \leftrightarrow A_{i-1} A_{i} \varnothing_{i+1} \text { rate } \alpha / 2, i=1,2,3, \ldots, \\
A_{0} A_{1} \rightarrow \varnothing_{0} \varnothing_{1} \text { rate } \lambda / 2, \\
\varnothing_{0} A_{1} \rightarrow A_{0} \varnothing_{1} \text { rate } \lambda / 2 .
\end{gathered}
$$

Excepting the boundary terms, we see that the Hamiltonian has been mapped back onto itself. Furthermore, at the edge, the particles may only hop from site 1 to site 0 , but never the other way round. This means that we can forget about the zeroth site in exchange for allowing the processes $A_{1} A_{2} \leftrightarrow A_{1} \varnothing_{2}$ (with rate $\alpha / 2$ ), and $A_{1} \rightarrow \varnothing_{1}$ (with rate $\lambda / 2$ ). Hence we see that the new Hamiltonian $\tilde{\mathcal{H}}$ corresponds to the case where $\mu_{s} \neq 0$, with the DP processes $A \leftrightarrow A+A$ and $A$ $\rightarrow \varnothing$ generated on the boundary.

If we choose the initial condition to be an uncorrelated state with density $1 / 2$, denoted by $|1 / 2\rangle$, then the density at site $k, \rho_{k}(t)$, is given by

$$
\rho_{k}(t)=\left\langle\left|n_{k} \exp (-\mathcal{H} t)\right| 1 / 2\right\rangle .
$$

Following exactly the procedure in Refs. [28,39] [starting with insertions of the identity operator $\mathcal{D D}^{-1}$ into the righthand side of Eq. (50)], one can straightforwardly show that

$$
\rho_{k}(t)=\frac{1}{2}[1-\langle 0|\exp (-\tilde{\mathcal{H}} t)| k-1, k\rangle]
$$

where $\langle 0|$ is the vacuum state (with no particles), and $|k-1, k\rangle$ is the initial state with only two particles situated at sites $k-1$ and $k$. However, relation (51) is just what we wanted to prove: the left-hand side is the density at the $k$ th site, whereas the right-hand side is $1 / 2$ times the probability that a cluster initiated at $t=0$ by two particles at sites $k-1$ and $k$ has not yet died out by time $t$. According to our earlier analysis, for $\Delta<0$, the left-hand side should scale as $|\Delta|^{\beta_{\text {dens }}}$ (far from the wall) or $|\Delta|^{\beta_{1, \text { dens }}^{\mathrm{Sp}}}$ (close to the wall), and the right-hand side as $|\Delta|^{\beta_{\text {seed }}}$ (far from the wall) or $|\Delta|^{\beta_{1, \text { seed }}^{\mathrm{O}}}$ (close to the wall). Thus, at the line $D=\lambda+\alpha$, we have shown the desired result $\beta_{1 \text {,seed }}^{\mathrm{O}}=\beta_{1 \text {,dens }}^{\mathrm{Sp}}$ (and, of course, the bulk result $\beta_{\text {seed }}=\beta_{\text {dens }}$ ). We note that the bulk result was proven in Ref. [28], and a very similar result for $A+A$ $\rightarrow \varnothing$, was derived in Ref. [39] (connecting the O* and Sp* "transitions"). Using universality, we postulate that the equality between the two surface exponents is valid everywhere close to the transition line, and not just where $D=\lambda$ $+\alpha$.

It is now straightforward to derive the relation $\beta_{1, \text { seed }}^{\mathrm{Sp}}$ $=\beta_{1, \text { dens }}^{\mathrm{O}}$ (again at the line $D=\lambda+\alpha$ ). One simply starts off with the quantum Hamiltonian $\widetilde{\mathcal{H}}$, and then follows the same steps as above. $\widetilde{\mathcal{H}}$ can then be mapped back onto the starting Hamiltonian $\mathcal{H}$, meaning that the transformation is actually a duality transformation. A relation like that in Eq. (51) can then be derived, giving $\beta_{1, \text { seed }}^{\mathrm{Sp}}=\beta_{1, \mathrm{dens}}^{\mathrm{O}}$.

In summary, at the particular line in parameter space $D$ $=\lambda+\alpha$, we have derived some exponent equalities which are in full agreement with the simulations to be presented in Sec. VI. In particular, we see that we have mapped BARW at the special transition onto BARW at the ordinary transition in $1+1$ dimensions (and vice versa), a rather nontrivial procedure. This has allowed us to derive some results about the $\beta_{1}$ exponents (something which seems to be beyond the ability of the field-theoretic methods at present). Unfortunately, as is always the case with exact calculations, the result is only derived for one line in parameter space, and we have to rely on universality in order to claim that it is valid elsewhere close to the transition line. 


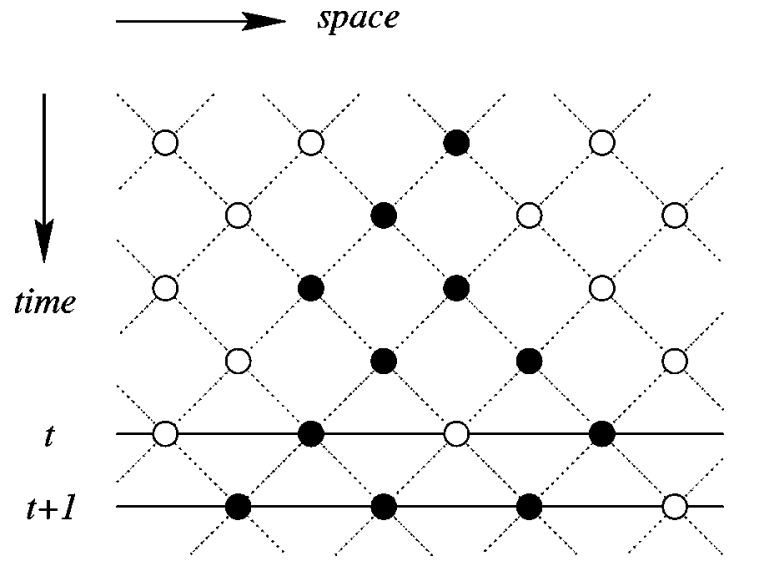

FIG. 6. Directed percolation in terms of the Domany-Kinzel model, where time flows vertically downward. Black sites are active $(A)$ and white ones inactive $(I)$. The state of each site at time $t+1$ depends on the states of the neighboring sites at time $t$.

\section{DPn AND BARW MODELS}

We will now briefly present the specific models and boundary conditions used in our numerical simulations. We begin with DP, include its generalization to DP2, and then comment on how we implement BARW. In all cases we include the specific boundary conditions and identify them according to the classification in Secs. III and IV.

For $d=1$, bond DP as well as site DP (for which the sites percolate instead of the bonds), are contained in the Domany-Kinzel model [40,41]. Each site can either be active or inactive and the probability for site $i$ to be updated to state $s_{i, t+1}$ at time $t+1$ is given by $P\left(s_{i, t+1} \mid s_{i-1, t}, s_{i+1, t}\right)$. See Fig. 6 for a typical lattice configuration, and Fig. 7 for the update rules.

The DP2 model has two symmetric absorbing states in which the system can be trapped. It is a special case of a generalized Domany-Kinzel model (DPn) introduced by Hinrichsen [12], where each site can be either active or in one of $n$ inactive states. For $n=1$ the update rules are identical to those of the Domany-Kinzel model in Fig. 7, but, for $n \geqslant 2$, the distinction between regions of different inactive states is preserved by demanding that they are separated by active ones. An example of a DP2 cluster is shown in Fig. 8(b), and we also show an ordinary DP cluster in Fig. 8(a) for comparison. In $1+1$ dimensions, DP2 belongs to the BARW universality class, and the update probabilities are given in Fig. 9.

The easiest way of introducing a boundary into DP and DP2 is simply to cut off the lattice. This is equivalent to introducing boundary sites which are forced to be in one of the inactive states. We will refer to this case as the inactive

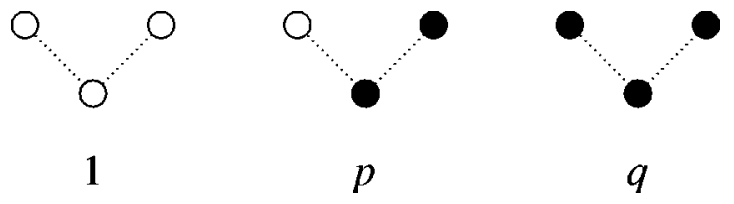

FIG. 7. Update probabilities for DP in terms of the parameters $0 \leqslant p, q \leqslant 1$, where we have $q=p(2-p)$ for bond DP and $q=p$ for site DP, respectively. Probabilities for the other configurations follow from left-right symmetry and from $P(A \mid \ldots)+P(I \mid \ldots)=1$.

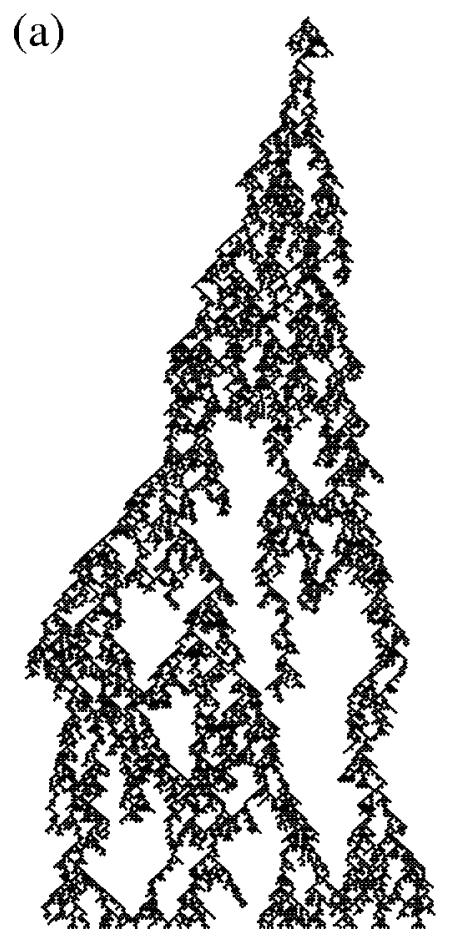

(b)

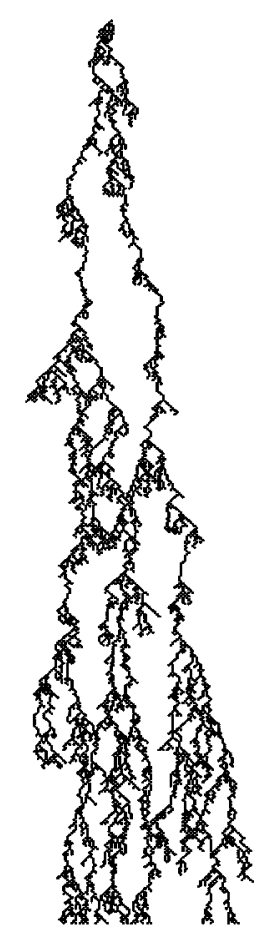

FIG. 8. (a) A DP cluster, and (b) a DP2 cluster, both grown from a single seed in the bulk.

boundary condition (IBC), and we choose inactivity of type 1 to the left of the boundary; see Fig. 10. Apart from imposing the state of these sites within the wall, the sites at the wall and those in the bulk are updated by the rules in Figs. 7 and 9.

Next we consider the reflecting boundary condition (RBC), where the wall acts like a mirror so that the sites within the wall are always a mirror image of those next to the wall; see Fig. 11. For DP2, one can see that there is a qualitative difference between the IBC and the RBC. For the latter, regions of type-2 inactivity can become trapped at the wall, and the only way for these regions to disappear is to wait for the cluster to return, whereas for the IBC such regions are never trapped.

We now consider the active boundary condition $(\mathrm{ABC})$,

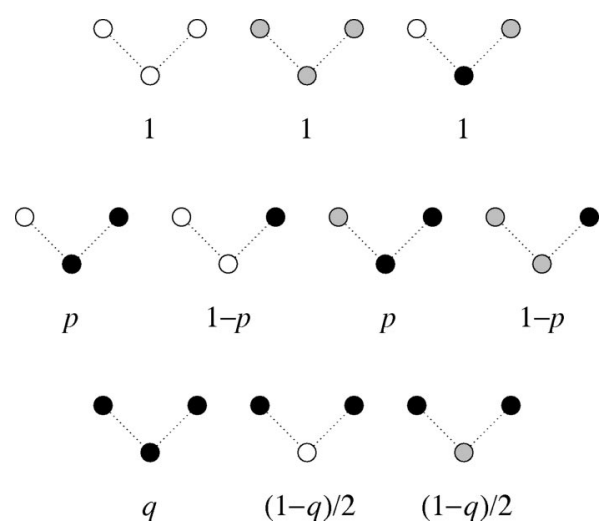

FIG. 9. Update probabilities for DP2: black sites are active $(A)$, whereas white and gray sites are in the inactive states $I_{1}$ and $I_{2}$, respectively. Probabilities for the other configurations follow from left-right symmetry and from $P(A \mid \ldots)+P\left(I_{1} \mid \ldots\right)+P\left(I_{2} \mid \ldots\right)$ $=1$. 


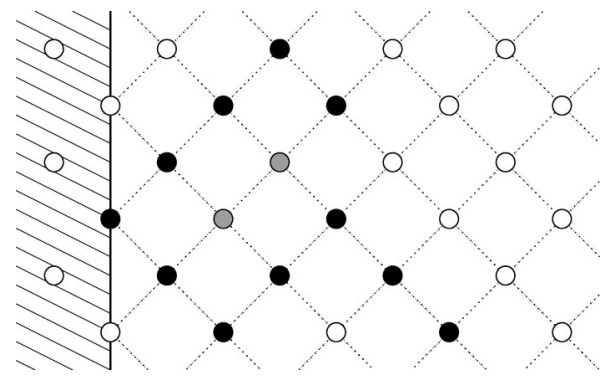

FIG. 10. DP2 with an inactive boundary condition (IBC), corresponding to the special $(\mathrm{Sp})$ universality class.

where the sites within the wall are forced to be active; see Fig. 12. In this case the cluster will never die completely as the wall will always be active and can always induce new clusters. Nevertheless, by defining the survival time of a cluster as the point in time when the system has no activity apart from within the wall itself, we can define the same exponents for the $\mathrm{ABC}$ as for the other boundary conditions. However, we have not studied this boundary condition in any detail but merely mention it here for completeness.

We can now discuss the relation between the above boundary conditions and our previous classification of the universality classes at the boundary for BARW in $1+1$ dimensions. The key feature is whether the symmetry between the two absorbing states in the bulk is preserved at the surface. In terms of the DP2 model, the IBC model respects this symmetry and hence belongs to the special $(\mathrm{Sp})$ universality class, whereas the RBC model does not respect this symmetry, and hence belongs to the ordinary $(\mathrm{O})$ universality class. Furthermore, the $\mathrm{ABC}$ model clearly belongs to the extraordinary $(E)$ universality class. Hence we see that by using the IBC, RBC, and ABC classification all the previously discussed boundary BARW transitions in $1+1$ dimensions can be accessed.

Furthermore, let us note that for DP the classification of the IBC and RBC is somewhat different. In $1+1$ dimensions DP probably does not support the special transition, and since no symmetry is broken by the RBC, both the IBC and $\mathrm{RBC}$ will belong to the ordinary transition universality class $[14,22]$.

We have also performed simulations for a lattice BARW model with IBC and RBC boundary conditions. For BARW we initially placed two particles at the two sites closest to the wall. The one-dimensional BARW model is then implemented with "dynamic branching," which means that the branching of one particle into three particles occurs ran-

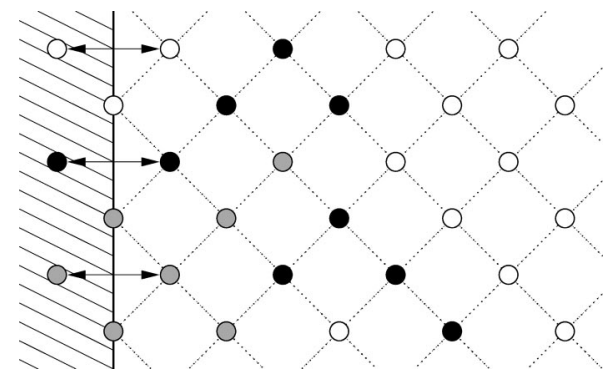

FIG. 11. DP2 with a reflecting boundary condition (RBC), corresponding to the ordinary $(\mathrm{O})$ universality class.

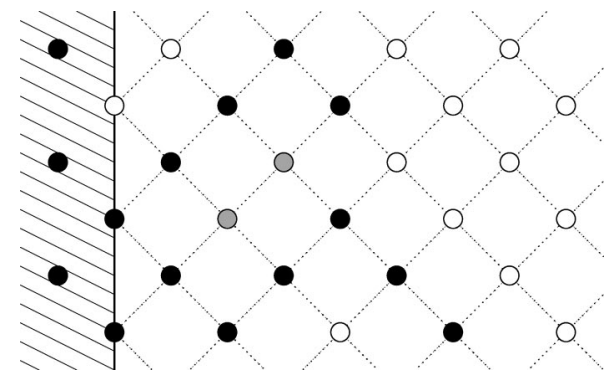

FIG. 12. DP2 with an active boundary condition (ABC), corresponding to the extraordinary (E) universality class.

domly to either the left or right of the particle [42]. The BARW model is expected to be in the same universality class as DP2, and our results are in agreement with this for both bulk and surface quantities (using the IBC and RBC). Hence, in Sec. VI, we will only discuss the results for DP2, since this is also the model to which we devoted most of our simulations.

\section{NUMERICAL RESULTS}

We studied DP2 in detail using Monte Carlo simulations in $1+1$ dimensions. The wall is placed at $i=0$, and we use an initial configuration with one active site at $i=0$, with the sites $i>0$ being in the inactive state $I_{1}$. Thus the absorbing state corresponds to the situation where all sites are in the inactive state $I_{1}$. The system is evolved according to the DP2 rules (see Fig. 9), and we typically average over $10^{5}$ independent clusters in order to reduce the error bars to a few percent. Using the notation of Fig. 9, we have carried out simulations for $q=p$ at the critical probability $p_{c}$, where we have used the estimate $p_{c}=0.5673$ [12].

In these simulations, starting from a seed on the wall, we measure the survival probability $P_{1}(t)$, the activity in the bulk $N_{1}(t)$ and at the wall $N_{1,1}(t)$, the average spread of the cluster $\left\langle x^{2}(t)\right\rangle$, and the probability $p_{1}(s)$ to have a cluster of size (mass) $s$, all at criticality [12,23]. Furthermore, by averaging over surviving clusters only (denoted by an over-line), we measure the surviving bulk activity $\overline{N_{1}}(t)$ and the surviving wall activity $\overline{N_{1,1}}(t)$, again starting from a seed on the wall.

First we performed simulations for DP2 without a wall, and obtained results for the exponents in complete agreement with those in Ref. [12]. Our results are listed in Tables I and II. There are several estimates available for the bulk exponent $\beta_{\text {dens }}\left(=\beta_{\text {seed }}\right)$ [43]. In the following we will use the estimate $\beta_{\text {dens }}=0.922(5)$ [44].

We now list some exponent relations used to extract the exponents from our numerical simulations [45]. All the relations given below are valid for both the IBC (special) and RBC (ordinary) DP2 transitions, and hence these labels will be suppressed from now on. The probability for a cluster grown from a seed on the wall still to be alive at time $t$ is given by Eq. (24). At criticality $(\Delta=0)$ it has the behavior

$$
P_{1}(t) \sim t^{-\delta_{1, \text { seed }},}
$$

with the exponent

$$
\delta_{1, \text { seed }}=\beta_{1, \text { seed }} / \nu_{\|} .
$$


TABLE I. Critical exponents obtained from our DP2 simulations. For comparison we also list the exponents for DP in the bulk and with an IBC wall $[15,18,47]$. The $\delta_{1, \text { seed }}$ exponent is obtained from Eqs. (56) and (57). Exponents without the " 1 " subscript refer to the bulk.

\begin{tabular}{|c|c|c|c|c|c|}
\hline & DP & DP (IBC) & DP2 & DP2 (IBC) & DP2 (RBC) \\
\hline$\overline{\kappa_{1}}$ & $0.47314(3)$ & $0.47314(3)$ & $0.288(5)$ & $0.287(2)$ & $0.285(2)$ \\
\hline$\delta_{\text {dens }}$ & $0.15947(3)$ & $0.15947(3)$ & $0.287(5)$ & $0.288(2)$ & $0.291(4)$ \\
\hline$\beta_{\text {dens }}$ & $0.27649(4)$ & $0.27649(4)$ & $0.922(5)$ & $0.93(1)$ & $0.94(2)$ \\
\hline$\kappa$ & $0.31368(4)$ & & $0.000(2)$ & & \\
\hline$\kappa_{1}$ & & $0.0496(3)$ & & $-0.354(2)$ & $-0.141(2)$ \\
\hline$\delta_{1, \text { seed }}$ & & $0.4235(3)$ & & $0.641(2)$ & $0.426(3)$ \\
\hline$\beta_{1, \text { seed }}$ & & $0.7338(1)$ & & $2.06(2)$ & $1.37(2)$ \\
\hline$\delta_{1, \mathrm{dens}}$ & & $0.4235(3)$ & & $0.415(3)$ & $0.635(2)$ \\
\hline$\beta_{1, \text { dens }}$ & & $0.7338(1)$ & & $1.34(2)$ & $2.04(2)$ \\
\hline $2 \chi$ & $1.26523(2)$ & & $1.150(5)$ & $1.150(3)$ & $1.152(3)$ \\
\hline$\chi$ & $0.63261(2)$ & & $0.575(3)$ & & \\
\hline$\nu_{\|}$ & $1.73383(3)$ & & $3.22(3)$ & & \\
\hline$\nu_{\perp}$ & $1.09684(2)$ & & $1.84(2)$ & & \\
\hline
\end{tabular}

Hence the probability of growing a cluster which lives ex-

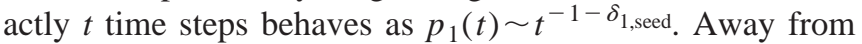
criticality it is straightforward to obtain the average cluster lifetime of finite clusters from Eq. (24). One obtains

$$
\langle t\rangle \sim|\Delta|^{-\tau_{1}}
$$

with the exponent

$$
\tau_{1}=\nu_{\|}-\beta_{1, \text { seed }}
$$

The average number of active sites at criticality, averaged over all clusters, is obtained by integrating the density (25) over space, and one arrives at

$$
N_{1}(t) \sim t^{\kappa_{1}}
$$

with

$$
\kappa_{1}=d \chi-\delta_{\mathrm{dens}}-\delta_{1, \mathrm{seed}},
$$

where we have introduced the cluster envelope or "roughness" exponent $\chi=\nu_{\perp} / \nu_{\|}(\equiv 1 / z)$, and the notation $\delta_{\text {dens }}$ $=\beta_{\text {dens }} / \nu_{\|}$. Note that Eq. (57) corresponds to the hyperscal- ing relation (30), a fact which follows from $\left\langle s_{1}(t)\right\rangle$ $=\int_{0}^{t} d t^{\prime} N_{1}\left(t^{\prime}\right)$, and the relation $\gamma_{1}=\nu_{\|}\left(1+\kappa_{1}\right)$. By integrating the density on the wall [Eq. (26)], we obtain the average number of active sites at criticality on the wall

$$
N_{1,1}(t) \sim t^{\kappa_{1,1}}
$$

with

$$
\kappa_{1,1}=(d-1) \chi-\delta_{1, \text { dens }}-\delta_{1, \text { seed }},
$$

and where $\delta_{1, \text { dens }}=\beta_{1 \text {,dens }} / \nu_{\|}$. Note also that Eq. (59) corresponds to the hyperscaling relation (32) at criticality, since $\left\langle s_{1,1}(t)\right\rangle=\int_{0}^{t} d t^{\prime} N_{1,1}\left(t^{\prime}\right)$, and $\gamma_{1,1}=\nu_{\|}\left(1+\kappa_{1,1}\right)$.

Alternatively, by averaging only over clusters which survive to infinity (denoted by an over-line), we obtain

$$
\overline{N_{1}}(t) \sim t^{\overline{\kappa_{1}}},
$$

where

$$
\overline{\kappa_{1}}=d \chi-\delta_{\mathrm{dens}} .
$$

TABLE II. Critical exponents for the cluster lifetime [Eq. (52)] and mass distributions [Eqs. (68), (69), (72), and (73)]. For comparison we also list the exponents for DP in the bulk and with an IBC wall $[15,18,47]$. We also give the exponents for the average lifetime [Eq. (54)], and average cluster sizes [Eqs. (29) and (31)], obtained from the scaling relations.

\begin{tabular}{lccccc}
\hline \hline & DP & DP (IBC) & DP2 & DP2 (IBC) & DP2 (RBC) \\
\hline$\delta_{\text {seed }}$ & $0.15947(3)$ & & $0.290(5)$ & & \\
$\delta_{1, \text { seed }}$ & & $0.4235(3)$ & & $0.646(3)$ & $0.425(3)$ \\
$\mu$ & $1.10825(2)$ & & $1.225(5)$ & & \\
$\mu_{1}$ & & $1.2875(2)$ & & $1.500(3)$ & $1.336(3)$ \\
$\mu_{1,1}$ & $1.18972(6)$ & $1.7337(2)$ & $1.408(5)$ & $2.05(5)$ & $2.15(5)$ \\
$\tau$ & $1.45734(7)$ & & $2.30(3)$ & & $1.85(4)$ \\
$\tau_{1}$ & & $1.0002(3)$ & & & \\
$\gamma$ & $2.27769(4)$ & & $3.22(5)$ & $2.08(4)$ & $(<7)$ \\
$\gamma_{1}$ & $1.18085(4)$ & $0.2664(3)$ & $1.38(3)$ & $(<0)$ & $(<0)$ \\
$\gamma_{1,1}$ & & & & & \\
\hline \hline
\end{tabular}


The activity on the wall for surviving clusters reads

$$
\left.\overline{N_{1,1}(} t\right) \sim t^{\overline{\kappa_{1,1}}}
$$

with the exponent

$$
\overline{\kappa_{1,1}}=(d-1) \chi-\delta_{1, \mathrm{dens}} .
$$

Simulations in $1+1$ dimensions thus directly yield $\delta_{1 \text {,dens }}$. The average position of activity follows from Eq. (25),

$$
\left\langle x^{2}\right\rangle \sim t^{2 \chi}
$$

where $x$ is the distance from the seed and the average is taken over all active points at a given time.

For further confirmation of our numerical data we also considered the cluster size distributions at criticality. In the bulk the typical cluster size $s$ of finite clusters scales as volume times density, i.e.,

$$
s \sim \xi_{\perp}^{d} \xi_{\|} n(\Delta) \sim|\Delta|^{-1 / \sigma},
$$

with

$$
1 / \sigma=d \nu_{\perp}+\nu_{\|}-\beta_{\mathrm{dens}}
$$

From the lifetime survival distribution (52), it is then straightforward to obtain the probability $P_{1}(s)$ to have a cluster of size larger than $s$, for clusters started from a seed on the wall. Using the fact that the lifetime is set by the parallel correlation length, $t \sim \xi_{\|} \sim|\Delta|^{-\nu \|}$, we see that the typical cluster size and lifetime are connected by

$$
s \sim t^{1 / \nu_{\|} \sigma} .
$$

Hence we obtain $P_{1}(s) \sim P_{1}\left(t \sim s^{\nu \| \sigma}\right) \sim s^{-\beta_{1, \text { seed }} \sigma}$. Thus we eventually obtain the probability $p_{1}(s)$ to have a cluster of exactly size $s, p_{1}(s)=-d P_{1}(s) / d s$, with the result

$$
p_{1}(s) \sim s^{-\mu_{1}},
$$

where

$$
\mu_{1}=1+\frac{\beta_{1, \text { seed }}}{d \nu_{\perp}+\nu_{\|}-\beta_{\text {dens }}} .
$$

Similarly, the cluster size distribution on the wall due to a seed located at the wall can also be obtained. In this case the typical cluster size of finite clusters is

$$
s_{\text {wall }} \sim \xi_{\perp}^{d-1} \xi_{\|} n_{1}(\Delta) \sim|\Delta|^{-1 / \sigma_{1}},
$$

where

$$
1 / \sigma_{1}=(d-1) \nu_{\perp}+\nu_{\|}-\beta_{1, \text { dens }} .
$$

The resulting distribution reads

$$
p_{1,1}\left(s_{\text {wall }}\right) \sim s_{\text {wall }}^{-\mu_{1,1}},
$$

with

$$
\mu_{1,1}=1+\frac{\beta_{1, \text { seed }}}{(d-1) \nu_{\perp}+\nu_{\|}-\beta_{1, \text { dens }}} .
$$

Note also that many of the scaling expressions given above only apply exactly at bulk criticality. Away from that point one must also include a scaling function. For example, Eq. (52) is replaced by $P_{1}(t, \Delta)=|\Delta|^{\beta_{1, \text { seed }}} F\left(t /|\Delta|^{-\nu} \|\right)$, and Eq. (68) is replaced by $p_{1}(s, \Delta)=|\Delta|^{\mu_{1} / \sigma} G\left(s /|\Delta|^{-1 / \sigma}\right)$.

In Tables I and II we list our estimates for the critical exponents for DP2. Our results are in complete accordance with our theoretical analysis: bulk exponents are unaltered whereas the wall introduces two separate surface exponents. We have also carried out bulk and surface simulations for $\Delta<0$, and confirmed that our data can be collapsed according to an appropriate survival probability scaling function [see Eq. (24) for the surface case], using our exponent estimates. This numerically confirms the validity of the relation $\delta=\beta / \nu_{\|}$for the bulk, as well as the analogous relations for both sets of surface exponents [46].

By using the explicit definitions of the IBC and RBC, we can deduce some further properties of the $\beta_{1 \text {,seed }}$ and $\beta_{1 \text {,dens }}$ exponents. There will be more activity next to the wall for the IBC than for the RBC, since the latter can have regions of $I_{2}$ located at the wall. Once created, these $I_{2}$ regions will survive until the activity returns to the wall. Thus it follows that $\beta_{1 \text {,dens }}^{\mathrm{IBC}} \leqslant \beta_{1 \text {,dens }}^{\mathrm{RBC}}$. On the other hand, the existence of these $I_{2}$ regions implies that the survival probability (24) for the $\mathrm{RBC}$ will be greater than for the IBC, leading to $\beta_{1, \text { seed }}^{\mathrm{IBC}}$ $\geqslant \beta_{1, \text { seed }}^{\mathrm{RBC}}$. Note that both our simulations and our previous exact calculations show that $\beta_{1 \text {,seed }}+\beta_{1 \text {,dens }}$ is the same for both the RBC and IBC. Using a hyperscaling relation [like that in Eq. (32)], this implies that $\gamma_{1,1}^{\mathrm{IBC}}=\gamma_{1,1}^{\mathrm{RBC}}$ (although both exponents defined in this way are negative). We have also studied several other boundary conditions, and found that these give the same scaling behavior as either the RBC or IBC depending on whether the above-mentioned $I_{2}$ regions can disappear only at the wall or also in the bulk.

We can obtain an interesting exponent relation for the RBC transition by assuming that the survival probability is dominated by the return to the wall of the cluster-envelope, which leads to [15]

$$
\delta_{1, \text { seed }}^{\mathrm{RBC}}=1-\chi
$$

in agreement with our simulation results for the RBC. Qualitatively, this means that the $I_{2}$ regions located at the wall determine the scaling, since they can only disappear when the activity returns to the wall. Note that a relation of this kind clearly fails for the IBC transition. Furthermore, if the cluster lifetime is defined to be the return time of the clusterenvelope (i.e., the return time of the rightmost active site) to the initial point, then we expect clusters defined in this way to have a lifetime distribution exponent $\delta_{1, \text { seed }}$ given by Eq. (74). This prediction is in agreement with the simulations in Ref. [9], where various models in the DP and BARW classes were studied with cluster lifetimes defined in the way described above.

For DP it has been customary for some time to investigate whether the critical exponents can be fitted by simple rational numbers [47]. Such a fitting has also been tried for bulk BARW with the following guesses in $1+1$ dimensions: $\kappa$ $=\chi-2 \delta=0$ and $\chi=4 / 7$ [5]. These estimates lead immediately to $\delta=2 / 7$ ( and $\beta / \nu_{\perp}=1 / 2, \gamma=\nu_{\|}$). It is intriguing to note that our numerical results for DP2 also suggest that 
$\mu_{1}=3 / 2$ for the IBC and $4 / 3$ for the RBC. From Eq. (69), it then follows that $\delta_{1 \text {,seed }}=9 / 14$ for the IBC and $3 / 7$ for the $\mathrm{RBC}$. We would need one more relation in order to obtain the last independent exponent, which we can take to be $\nu_{\|}$. In fact, we observe numerically that the relation $2 \nu_{\|}$ $-\beta_{1, \text { seed }}-\beta_{1, \text { dens }}=3$ is valid to within $1 \%$. From these observations the remaining DP2 exponents follow: $\beta_{\text {dens }}$ $=\beta_{\text {seed }}=12 / 13, \nu_{\|}=42 / 13$, and $\nu_{\perp}=24 / 13$. Furthermore, $\beta_{1, \text { seed }}=27 / 13$ and $\beta_{1, \text { dens }}=18 / 13$ for the IBC, and vice versa for the RBC. However, at present we have no understanding of these possible exact values for the $(1+1)$-dimensional exponents. BARW is certainly not conformally invariant, and consequently until some theoretical framework is proposed to explain why these exponents could be rational numbers, numerical coincidence remains a distinct possibility.

\section{CONCLUSION}

In this paper we have presented a study of critical surface effects in systems with nonequilibrium phase transitions. In particular we have focused on the DP and BARW universality classes, where we have put forward a unified presentation involving mean field, scaling, field-theoretic, and exact methods. Furthermore, many of our theoretical conclusions have been backed up by large-scale Monte-Carlo simulations.

Nevertheless, there are still a number of open questions. In particular, our understanding of surface BARW in $1+1$ dimensions is hampered by the fundamental problems of the field theory, which mean that the boundary (and bulk) transitions occurring at $\sigma_{m}=\sigma_{m \text {, critical }}$ remain difficult to treat using RG methods. Furthermore, we have concentrated on the ordinary and special transitions in $(1+1)$-dimensional BARW - there will most likely also be interesting behavior for the extraordinary transition.

Finally, our most important result is the existence of two independent surface exponents: $\beta_{1, \text { dens }}$ and $\beta_{1, \text { seed }}$ for surface BARW (and DP2). This certainly distinguishes DP from BARW, since, for the former case, $\beta_{1, \text { seed }}=\beta_{1, \text { dens }}$. For the $(1+1)$-dimensional BARW case, on the other hand, we have used exact techniques to link the surface exponents at the ordinary and special transitions, giving $\beta_{1, \text { seed }}^{\mathrm{O}}=\beta_{1, \text { dens }}^{\mathrm{Sp}}$ and $\beta_{1, \text { seed }}^{\mathrm{Sp}}=\beta_{1, \text { dens }}^{\mathrm{O}}$. It would certainly be instructive to rederive these results from a field-theoretic perspective, but this is beyond the scope of the present paper.

\section{ACKNOWLEDGMENTS}

We would like to thank Tim Newman, Beate Schmittmann, and Uwe Täuber for very useful discussions. M.H. acknowledges support from the U.S. National Science Foundation through the Division of Materials Research, and is grateful for hospitality and financial support from the CATS group at the Niels Bohr Institute, where part of this work was performed. P.F. acknowledges support from the Swedish Natural Science Research Council. K.B.L. acknowledges support from the Carlsberg Foundation.
[1] For reviews of surface critical phenomena, see K. Binder, in Phase Transitions and Critical Phenomena, edited by C. Domb and J. L. Lebowitz (Academic Press, London, 1983), Vol. 8; H. W. Diehl, in Phase Transitions and Critical Phenomena, edited by C. Domb and J. L. Lebowitz (Academic Press, London, 1986), Vol. 10; Int. J. Mod. Phys. B 11, 3593 (1997).

[2] W. Kinzel, in Percolation Structures and Processes, edited by G. Deutscher, R. Zallen, and J. Adler, Annals of the Israel Physical Society Vol. 5 (Adam Hilger, Bristol, 1983).

[3] R. Dickman, in Nonequilibrium Statistical Mechanics in One Dimension, edited by V. Privman (Cambridge University Press, Cambridge, 1997).

[4] H. Takayasu and A. Yu. Tretyakov, Phys. Rev. Lett. 68, 3060 (1992).

[5] I. Jensen, Phys. Rev. E 50, 3623 (1994).

[6] J. Cardy and U. C. Täuber, Phys. Rev. Lett. 77, 4780 (1996); J. Stat. Phys. 90, 1 (1998).

[7] P. Grassberger, F. Krause, and T. von der Twer, J. Phys. A 17, L105 (1984); P. Grassberger, ibid. 22, L1103 (1989).

[8] M. H. Kim and H. Park, Phys. Rev. Lett. 73, 2579 (1994); H. Park, M. H. Kim, and H. Park, Phys. Rev. E 52, 5664 (1995).

[9] W. M. Hwang, S. Kwon, H. Park, and H. Park, Phys. Rev. E 57, 6438 (1998).

[10] W. M. Hwang and H. Park, Phys. Rev. E 59, 4683 (1999).

[11] N. Menyhárd and G. Ódor, J. Phys. A 29, 7739 (1996).

[12] H. Hinrichsen, Phys. Rev. E 55, 219 (1997).

[13] H. K. Janssen, B. Schaub, and B. Schmittmann, Z. Phys. B 72, 111 (1988).
[14] P. Fröjdh, M. Howard, and K. B. Lauritsen, J. Phys. A 31, 2311 (1998).

[15] K. B. Lauritsen, K. Sneppen, M. Markošová, and M. H. Jensen, Physica A 247, 1 (1997).

[16] M. A. de Menezes and C. F. Moukarzel, Phys. Rev. E 60, 5699 (1999).

[17] E. Carlon, M. Henkel, and U. Schollwöck, Eur. Phys. J. B 12, 99 (1999).

[18] J. W. Essam, A. J. Guttmann, I. Jensen, and D. TanlaKishani, J. Phys. A 29, 1619 (1996).

[19] I. Jensen, J. Phys. A 32, 6055 (1999).

[20] H. Hinrichsen and H. M. Koduvely, Eur. Phys. J. B 5, 257 (1998).

[21] C.-C. Chen, H. Park, and M. den Nijs, Phys. Rev. E 60, 2496 (1999).

[22] K. B. Lauritsen, P. Fröjdh, and M. Howard, Phys. Rev. Lett. 81, 2104 (1998).

[23] P. Grassberger and A. de la Torre, Ann. Phys. (N.Y.) 122, 373 (1979).

[24] J. L. Cardy and R. L. Sugar, J. Phys. A 13, L423 (1980).

[25] H. Janssen, Z. Phys. B 42, 151 (1981).

[26] P. Grassberger and K. Sundermeyer, Phys. Lett. B 77, 220 (1978).

[27] Note that the convention used for $\Delta$ here is different from that in Ref. [22].

[28] K. Mussawisade, J. E. Santos, and G. M. Schütz, J. Phys. A 31, 4381 (1998).

[29] J. L. Cardy, Scaling and Renormalization in Statistical Physics 
(Cambridge University Press, Cambridge, 1996).

[30] Note that the mean field surface density exponent $\beta_{1, \text { dens }}^{\mathrm{IBC}}$ quoted in footnote [22] of Ref. [22] is incorrect.

[31] For $\Delta>0$ we have $n_{s}=\left(3 \Delta_{s}^{2} / 2 D \lambda\right)-(3 \Delta / 2 \lambda)$, whereas for $\Delta<0$ we have $n_{s}=\left(3 \Delta_{s}^{2} / 2 D \lambda\right)-(3 \Delta / 2 \lambda)+\left(2 D^{2} \Delta^{3} / 9 \lambda \Delta_{s}^{4}\right)$ plus higher order terms.

[32] A. J. Bray and M. A. Moore, J. Phys. A 10, 1927 (1977).

[33] M. J. E. Richardson and Y. Kafri, Phys. Rev. E 59, R4725 (1999); Y. Kafri and M. J. E. Richardson, J. Phys. A 32, 3253 (1999).

[34] A. Drewitz, R. Leidl, T. W. Burkhardt, and H. W. Diehl, Phys. Rev. Lett. 78, 1090 (1997).

[35] L. Peliti, J. Phys. (France) 46, 1469 (1985).

[36] B. Lee, J. Phys. A 27, 2633 (1994).

[37] D. ben-Avraham, J. Chem. Phys. 88, 941 (1988).
[38] D. Considine and S. Redner, J. Phys. A 22, 1621 (1989).

[39] G. M. Schütz, Z. Phys. B 104, 583 (1997).

[40] E. Domany and W. Kinzel, Phys. Rev. Lett. 53, 311 (1984).

[41] W. Kinzel, Z. Phys. B 58, 229 (1985).

[42] S. Kwon and H. Park, Phys. Rev. E 52, 5955 (1995).

[43] I. Jensen, J. Phys. A 30, 8471 (1997).

[44] D. Zhong and D. ben-Avraham, Phys. Lett. A 209, 333 (1995).

[45] Note that some of our exponent definitions in this section differ from those sometimes found in the literature. In particular our $\kappa$ exponents are sometimes called $\eta$, and the dynamic exponent $z$ is sometimes redefined as $z \rightarrow 2 / z$.

[46] Technical difficulties have so far prevented a field-theoretic derivation of these kinds of relations for BARW [6].

[47] I. Jensen, J. Phys. A 29, 7013 (1996). 